\title{
A Photometric and Spectroscopic Study of the Cataclysmic Variable ST LMi during 2005-2006, ${ }^{2}$
}

\author{
S. Kafka ${ }^{1,2,3}$ \\ S.B. Howell ${ }^{3,4,5}$ \\ R.K. Honeycutt ${ }^{3,6,7}$ \\ and \\ J.W. Robertson $3,8,9$
}

\begin{abstract}
We present orbit-resolved spectroscopic and photometric observations of the polar ST LMi during its recent low and high states. In the low state spectra, we report the presence of blue and red satellites to the $\mathrm{H} \alpha$ emission line; the velocities and visibility of the satellites vary with phase. This behavior is similar to emission line profile variations recently reported in the low state of AM Her, which were interpreted as being due to magnetically-confined gas motions in large loops near the secondary. Our low-state spectroscopy of ST LMi is discussed in terms of extreme chromospheric activity on the secondary star. Concurrent photometry indicates that occasional low-level accretion may be present, as well as cool regions on the secondary near $\mathrm{L}_{1}$ Furthermore, we report a new "extreme low-state" of the system at V 18.5 mag.

Our orbital high-state spectroscopy reveals changes in the emission line profiles with orbital phases that are similar to those reported by earlier high-state studies. The complicated emission line profiles generally consist of two main components. The first
\end{abstract}

\footnotetext{
${ }^{1}$ CTIO/NOAO, Casilla 603, La Serena, Chile.

${ }^{2}$ E-mail: skafka@noao.edu

${ }^{3}$ Visiting Astronomer, Kitt Peak National Observatory, National Optical Astronomy Observatory, which is operated by the Association of Universities for Research in Astronomy, Inc. (AURA) under cooperative agreement with the National Science Foundation.

${ }^{4}$ WIYN Observatory \& NOAO, P.O. Box 26732, 950 N. Cherry Ave., Tucson, AZ 85719

${ }^{5}$ E-mail: howell@noao.edu

${ }^{6}$ Indiana University, Astronomy Department, 319 Swain Hall West, Bloomington, IN 47405

${ }^{7}$ E-mail: honey@astro.indiana.edu

${ }^{8}$ Arkansas Tech Univ., Dept. of Physical Sciences, 1701 N. Boulder, Russellville, AR 72801-2222

${ }^{9}$ E-mail: jeff.robertson@atu.edu
} 
has radial velocity variations identical to that of the major emission $\mathrm{H} \alpha$ component seen in the low state. The second is an additional red-shifted component appearing at the phases of maximum visibility of the accreting column of the white dwarf; it is interpreted as being due to infall velocities on the accreting magnetic pole of the white dwarf. At the opposite phases, an extended blue emission wing appears on the emission line profiles. We confirm the presence of a broad absorption feature near $6275 \AA$ which has been previously identified as Zeeman $\sigma^{-}$absorption component to $\mathrm{H} \alpha$. This feature appears at just those phases when the accretion pole region is mostly directly visible and most nearly face-on to the observer.

Subject headings: binaries: close - stars: stars: general — stars: individual (ST LMi),stars: magnetic fields, stars: activity

\section{Introduction}

Cataclysmic Variables (CVs) are short orbital period (hours to days) interacting binaries containing a white dwarf (WD) primary and a K-M (or later) dwarf mass losing secondary star. It is generally accepted that mass transfer occurs as the secondary overflows its Roche lobe and matter falls toward the primary star through the inner Lagrangian point (L1). The future of the accretion stream is then determined by the magnetic field of the WD: in magnetic systems the stream is channeled toward the magnetic poles of the primary along its magnetic field lines; in non-magnetic systems a hot accretion disk is formed around the primary, replenished by continuous mass transfer. The abundance of CV properties is reviewed in Warner (1995) and will not be repeated here. Among the various subcategories, there is one which includes both disk and magnetic CVs with large (up to $5 \mathrm{mag}$ ) apparently random drops in the optical brightness; these are known as VY Scl stars. Although there is no well-accepted explanation for the cause of these low states, it is clear that accretion from the secondary star is significantly diminished, often exposing the two stellar components and providing a unique opportunity to study their photospheres. Sadly, low states in CVs are erratic in occurrence and duration, making observational study a challenge. Therefore, we generally rely on photometric monitoring efforts by professional or amateur astronomers in order to ascertain when a given system is in a low state.

Recently, low state observations of the magnetic CV prototype, AM Her, revealed short outburst-like events in the optical light curves of the system which were attributed to flares (ac-

\footnotetext{
${ }^{1}$ Based on observations from the WIYN Observatory, which is a joint facility of the University of WisconsinMadison, Indiana University, Yale University, and the National Optical Astronomy Observatories, USA.

${ }^{2}$ Based on observations obtained with the Mayall $4 \mathrm{~m}$ telescope at Kitt Peak National Observatory, a division of the National Optical Astronomy Observatory, which is operated by the Association of Universities for Research in Astronomy Inc., under cooperative agreement with the National Science Foundation
} 
tivity) on the secondary star (Kafka et al. 2005a). Later work (Kafka et al. 2005b) revealed components of the $\mathrm{H} \alpha$ emission line arising from the vicinity of the secondary star. These features were interpreted as being due to stellar activity on the secondary and their study provides a new method to explore the extent and variability of magnetic structures on the low-mass donor star. Stellar activity on the secondary in CVs has been invoked to explain a plethora of phenomena, such as long-term light curve variations (Bianchini 1988) and high/low states (Livio and Pringle 1994). However, correlation of such phenomena with stellar activity has yet to be fully explored. In AM Her, prominence-like loops with gas velocities of $\sim 300 \mathrm{~km} / \mathrm{sec}$ persisted for more than three years (Kafka, Honeycutt \& Howell 2006), suggesting that they might be present even when the system is in a high state, albeit masked by accretion flux (Kafka et al. 2005b). Recently the polar VV Pup exhibited similar structures in the $\mathrm{H} \alpha$ emission line during its low state (Mason et al. 2007). In VV Pup the $\mathrm{H} \alpha$ emission structures were highly variable on timescales of several weeks.

ST LMi (CW 1103+254) was introduced as a magnetic CV in the work of Stockman et al. (1983), in which linear and circular polarization varied with the $114 \mathrm{~min}$ orbital period of the system. Several high-state emission line components (Stockman et al. 1983; Bailey et al. 1985) have been attributed to infall velocities and to features arising near the secondary star. Most studies (Stockman et al. 1983; Schmidt, Stockman \& Grandi 1983; Bailey et al. 1985; Cropper 1986; Cropper \& Horne 1994, Stockman \& Schmidt 1996) have concluded that the system is normally a one-pole accretor, in which the active pole is self-eclipsed by the limb of the white dwarf for a major part of the orbit. Peacock et al. (1992) reported polarimetric evidence for accretion onto a second pole at times. Schmidt, Stockman and Grandi (1983) provided a geometrical model of the system, according to which the secondary star slightly lags the single accreting magnetic pole. Using near-IR data, Ferrario, Bailey \& Wickramasinghe (1993) modeled the high state cyclotron emission from the active pole and derived a magnetic field strength of $11.5 \pm 0.5 \mathrm{MG}$.

Since 1990, ST LMi has been a part of the long-term monitoring program of a 0.41-m automated telescope located in central Indiana (RoboScop 3 ). The 1990-2004 light curve of the system, which appeared in Kafka \& Honeycutt 2005 (KH05), revealed a 5-year long low state (1992-1997), followed by $\sim 6$ years of high state (Fig 1). During the high state, the light curve of ST LMi shows a $\sim 1.5$ mag orbital modulation caused by the self-eclipse of the single accreting pole by the white dwarf (Stockman \& Schmidt 1996). During the low states, the RoboScope light curve showed that ST LMi usually reached a quiescent magnitude of 17.5 with an approximate sinusoidal modulation of $\sim 0.2$ mag (KH05).

In this paper, we present a photometric and spectroscopic study of the system during 2005 and 2006. In Section 2 we describe our data and data reductions, in Section 3 we discuss the results, while in Section 4 we compare these results to the behavior of other polars in the low state. Our conclusions are summarized in Section 5. The results of this study show that the secondary star in ST LMi is a highly active star and behaves in a similar manner as those observed in a small but

\footnotetext{
${ }^{3}$ Honeycutt et al. 1994 and refs therein
} 
growing sample of polar mass donors observed during low states.

\section{Observations and Reductions}

\subsection{Photometry}

In the top panel of figure 1 we have combined our V-band photometry of ST LMi from various sources. The data prior to 2004-Nov are RoboScope data from KH05. RoboScope continued to collect photometry of ST LMi until 2005-Mar, which is also included in figure 1; after 2005-Mar RoboScope ceased operation (temporarily we hope). The RoboScope data were processed/reduced through a pipeline that performs aperture photometry and extracts light curves of all the stars in the field based on the method of incomplete ensemble photometry (Honeycutt 1992).

The photometry later than 2005-Mar in figure 1 is mostly from the WIYN 0.91-m telescope at Kitt Peak. Processing of the non-RoboScope images followed standard IRAF 4 procedures, using aperture photometry for the extraction of instrumental magnitude. We used stars from Henden \& Honeycutt (1995) as secondary standards. Much (but not all) of this 0.91-m photometry consists of sequences of exposures covering one orbital period; these sequences are grouped into several observing runs, designated A-D (figure 1 and Table 1). Figures 2-4 show our phased photometric light curves of ST LMi, using only the data from figure 1 that is continuous for $\sim 1$ orbit. In this paper we have adopted an orbital ephemeris which uses the period from Cropper (1986) and a spectroscopic zeropoint (or epoch) from Howell et al. (2000). The spectroscopic epoch is based on IR observation of photospheric absorption lines from the secondary star and phase zero marks the time of inferior conjunction of the secondary. For reference, phase zero for our ephemeris corresponds to phase 0.17 on the polarimetric ephemeris of Cropper (1986), thereby placing the expected location of the observer's most direct view of the active accretion pole region near our phase 0.83. The A-D designations shown in fig. 1, and listed in Table 1, are used as appropriate in figures 2-3. We show phased RoboScope light curves in fig. 4.

\subsection{Spectroscopy}

Our spectroscopic data were obtained in four different observing runs during the interval 2005Jun to 2006-May (indicated in figure 1, bottom). During runs 2005-Jun (one night) and 2006-Feb (two nights) the HYDRA multi-object fiber spectrograph (MOS) on the WIYN $5.5-\mathrm{m}$ telescope

\footnotetext{
${ }^{4}$ IRAF is distributed by the National Optical Astronomy Observatories, which are operated by the Association of Universities for Research in Astronomy, Inc., under cooperative agreement with the National Science Foundation.

${ }^{5}$ The WIYN Observatory is a joint facility of the University of Wisconsin-Madison, Indiana University, Yale University, and the National Optical Astronomy Observatories, USA.
} 
was used. At WIYN we employed the 600 line $\mathrm{mm}^{-1}$ grating in first order, blazed at $7500 \AA$; the spectral coverage was $\sim 5500-8000 \AA$, with a resolution of $\sim 3 \AA$. A number of non-assigned fibers were placed at random locations to serve for sky subtraction, and a $\mathrm{CuAr}$ lamp was used for wavelength calibration. The R-C Spectrograph on the 4-m KPNO telescope was used to acquire two spectra of ST LMi in 2005-Dec, and to obtain a sequence of spectra covering a little more than one orbit in 2006-May. For the 2005-Dec spectra we used the KPC-007 grating and the GG-385 order separation filter. The spectral coverage was $\sim 5500-7500 \AA$ with a resolution of $\sim 3 \AA$. For the 2006-May data we used grating KPC-24 in second order with a 1" slit to match the seeing. This combination provided $\sim 1000 \AA$ coverage at $1.3 \AA$ resolution. The 2006 -May night was clear with photometric conditions and stable seeing, allowing extraction of good quality relative photometric information from these spectra. We produced a synthetic light curve using the fluxed spectra from this night of high state spectroscopy (figure 3 bottom right, see $\S 4.2$ ). An instrumental magnitude was extracted from the reduced 4-m spectra using imstat to sum the counts in rectangular windows (including both lines and continuum) for star and sky. The length of each window was $\sim 80 \%$ of the length of the spectrum, yielding an effective wavelength approximately midway between $\mathrm{V}$ and R. Exposure times for all our spectra ranged between 300 and $1800 \mathrm{sec}$, depending on the weather conditions (Table 1).

Data processing and reduction employed standard IRAF procedures such as twodspec with wavelength calibration accomplished via the use of arc lamp exposures obtained near in time and position to our stellar exposures. For the R-C spectrograph data, we extracted the 2-d images, reduced them in the usual fashion, flux calibrated them using the spectrophotometric standard star GD140, and produced final 1-d spectra. No corrections were made for atmospheric extinction as the slit was kept at the parallactic angle and the total range of airmass was small and never larger than 1.6. The usual telluric absorption features remain in all the final spectra. For Hydra, we performed the additional steps to allow correction for scattered light and the more complex sky

subtraction necessary for a fiber spectrograph. Partly cloudy weather conditions prevailed during all our runs with the exception of the 2006-May observations.

\section{Photometric Results}

The photometric coverage in figure 1 is not continuous during 2005-2006 (due to the untimely failure of RoboScope) but the available photometry does show that ST LMi was in a nearlycontinuous low state during most of our photometric and spectroscopic observations. In 2005-2006, the most common basal magnitude in the low state appears to be $\sim 17.5$ (bottom panel of figure 1); consistent with the brightness during the 1992-1996 low state recorded by RoboScope (top panel of figure 1). However, the photometric sequence of 2006-Feb-12 (UT) (sequence C1 in Table 1) shows that the system reached $\mathrm{V} \sim 18.0-18.4$, significantly fainter than other data points in figure 1 . The 0.91-m exposures showing this unusually faint state were acquired using the same equipment, and were reduced using the same procedures as our other photometric observations near this time (see 
table 1) which show the system at $\mathrm{V} \sim 17.5$. We therefore conclude that this unusually low state is real. Although it appears inconsistent with the rest of the RoboScope data (which never showed such a state over 13 years), the RoboScope magnitude limit is about 17.8; therefore fainter events would escape detection. Although unusual, the presence of an "extreme-low state" is not unique to ST LMi; VV Pup, for example, was also observed to have two different low state levels (Mason et al. 2007). This extreme low state, allows for an interesting experiment for ST LMi: assuming that the extreme low state (mean $\mathrm{V}=18.2 \mathrm{mag}$ ) represents a time where activity and accretion temporarily stop then the observed light in $\mathrm{V}$ should be dominated by the white dwarf alone. For a DA WD, $\mathrm{M}_{V}=12.5$. This gives a distance for ST LMi of $138 \mathrm{pc}$. This is very similar to the distance of $136 \mathrm{pc}$ derived by Bailey et al. (1985) using the K surface brightness for an M5 main sequence star and a secondary mass of $0.18 \mathrm{M}_{\text {sun }}$.

The low state orbital light curves from our photometric monitoring are shown in figures 2 and 3. Light curves A1-A5 show a 0.3-0.8 mag "bump" near phase 0.8, the phase at which we most directly view the main accretion region on the magnetic white dwarf. Although the mean magnitude of these sequences was about at the "normal" low state brightness, the observed bump in the light curves suggest that some low-level variable accretion (likely wind accretion) was taking place in 2005-late Feb/early March. In contrast, the low state orbital light curves for 2005-Jun and 2006-Feb (B1-B4, D1) appear consistent with the 1992-1996 RoboScope low-state orbital light curve shown in fig. 4, bottom panel). These low state light curves are essentially featureless with (at best) only a $\sim 0.1 \mathrm{mag}$ sinusoidal variation superposed on $\sim 0.2 \mathrm{mag}$ random variations. Note that even the random variation is largely missing for the low-state orbital light curve for UT 2006Feb-21 (fig. 3, D1). In 2005-March-1 (UT) we monitored ST LMi (A2) using an R filter. Figure 3 (right, top) shows our phased differential R band light curve with an arbitrary zero point. Again we see an orbital bump during this low state observation but with a larger amplitude than observed in $\mathrm{V}$ at nearly the same time (fig. 2). Even with a slightly variable amplitude from night to night, the larger amplitude bump observed in $\mathrm{R}$ compared with $\mathrm{V}$ during the low state agrees with previous high state observations of ST LMi showing that it becomes redder near phase 0.8 . We will see below that for ST LMi, the red color at this phase is due to the contribution of the cyclotron continuum to the overall flux, a contribution that outshines the other components during this phase interval.

It is of particular importance to ascertain the photometric state at the time of the spectroscopic observations, using both the brightness of the system and the orbital light curve. For the 2005Jun-17 WIYN spectra, simultaneous photometry (figure 2, sequence B2) shows V 17.5 magnitude, with little or no orbital modulation. Therefore the system was in the normal low state, with no evidence of accretion. For the 2005-Dec-31 4-m spectra we have V-band measures on two nights, 7 and 8 nights preceding the spectroscopy, from Tenagra Observatory 6 , showing ST LMi at V 17.3, again indicating a normal low state. During the 2006-Feb-21/22 (UT) WIYN spectroscopy, we have simultaneous WIYN 0.91-m photometry showing the system at V 17.5. The orbital light curve

\footnotetext{
${ }^{6}$ http://www.tenagraobservatories.com
} 
for 2006-Feb-22 (UT) (sequence D1, figures 1 and 4) is very "quiet", with no significant orbital variations. Therefore the system showed no evidence of accretion for 2006-Feb-21 (UT) (based on its brightness) nor on 2006-Feb-22 (UT) (based on both the brightness and on the flat orbital light curve). The 2006-Feb-21/22 data is the nearest photometric measurement to our 2006-May-19 4-m spectroscopy available to us. In order to better determine the photometric state for the 2006-May spectroscopy we produced a relative light curve using the summed flux in the 4-m spectra (see section 2.2 for details). The transparency and seeing on this night were very good and stable, resulting in a quantitatively good light curve (figure 3, bottom right) at an effective wavelength which is approximately mid-way between $\mathrm{V}$ and $\mathrm{R}$, and has an arbitrary zeropoint. This light curve has a conspicuous $1.7 \mathrm{mag}$ "bump" near phase 0.8 , similar to that seen in high-state photometry such as the RoboScope high-state orbital light curve (top panel, figure 47 ). The bump at phase 0.8 is attributed to accretion onto the white dwarf pole as seen most directly by the observer. While some low state photometry shows residual accretion, likely due to wind accretion (e.g., sequences A1-A5, fig. 2) the amplitude and consistency of the bump seen in the 2006-May light curve (fig. 3 ) is similar to the RoboScope photometry high state light curve (fig. 4, top). Thus, it seems that during our 2006-May spectroscopy ST LMi was probably no longer in the low state; this will be discussed in Sec. 4.2.

In summary, we conclude that the orbit-resolved WIYN spectroscopy of 2005-Jun and 2006Feb were in the low state, whereas the orbit-resolved 4-m spectra of 2006-May were likely in the high state.

\section{Spectroscopic Results}

\subsection{Low State Spectra}

Figure 5 shows representative spectra from the three low-state spectroscopic runs in 2005-Jun, 2005-Dec, and 2006-Feb. All spectra were taken at a similar spectral resolution of $\sim 3 \AA$ and clearly show the $\mathrm{TiO}$ bandheads near $7050 \AA$ from the secondary star, confirming our earlier conclusion that any accretion luminosity was very low at these epochs. The $\mathrm{H} \alpha$ emission line has considerable structure, as shown in the enlarged inset plots, similar to the profiles of the H $\alpha$ line of AM Her (Kafka et al. 2005b; 2006) and VV Pup (Mason et al. 2007) during their recent low states.

Figures 6 and 7 show the $\mathrm{H} \alpha$ emission line profiles as a function of phase for two adjacent nights of WIYN spectroscopy 2006-Feb-21/22 UT. In both nights, the emission line occasionally develops a red satellite near phases 0.75-1.00 and a blue component to the $\mathrm{H} \alpha$ emission line for phases near 0.4 and 0.5. The complex emission line profiles have been fitted with Gaussian (in a manner identical to that described in Kafka et al. 2006 for AM Her) to determine their radial velocities (RVs). We

\footnotetext{
${ }^{7}$ the phasing of the folded light curves of ST LMi in KH05 (figure 5 in that paper) is in error because a wrong epoch for the ephemeris was mistakenly employed.
} 
have been conservative in choosing between noise in the profile and a real emission line component; we used satellites that exceeded the noise level by $2.5 \sigma$. The RV curves for the line components we judged to be reliable, are shown in figure 8 . Figure 8 also includes the RV of the central peak of the $\mathrm{H} \alpha$ line for the 2005-June (squares) and the 2005-December (triangles) data. The RVs of the satellites are plotted with open symbols; different colors correspond to different cycles. The RV phasing of the central emission line component of $\mathrm{H} \alpha$ indicates that it arises from the secondary star's side of the center of mass. The blue/red crossing is 0.05 phase units away from phase zero (which marks inferior conjunction of the secondary), but is consistent with the uncertainty of $\sim 0.1$ phase units quoted by Howell et al. (2000) for the time of conjunction in the ephemeris. The velocities and phase visibility of the occasional blue and red satellites in figure 8 are quite similar to that those reported for AM Her (Kafka et al. 2005, 2006) and VV Pup (Mason et al. 2007); they have been interpreted as arising from gas motions along two large magnetically-confined loops, perhaps similar to slingshot prominences.

Figure 9 (top) shows the low-state equivalent width of the full $\mathrm{H} \alpha$ emission line for the WIYN spectroscopy of 2006-02-21/22 vs orbital phase. For comparison, we reproduce the phased light curve (D1) of figure 3 on a expanded magnitude scale so as to see the structure of the lightcurve. The $\mathrm{H} \alpha$ emission line appears to be stronger between phases 0.1-0.5 (albeit with variable strength); this is the trailing side of the secondary star. Near the same phases the light curve appears to have a V 0.05 mag dip, which is repeated in two consecutive orbits. Since accretion was absent at this epoch, the light curve dip could be due to the presence of a dark starspot region on the secondary star, with the $\mathrm{H} \alpha$ brightening corresponding to associated chromospheric activity. This behavior suggests that magnetic activity (spots) near the L1 point may indeed be connected to the occurrence of the low states, in agreement with the Livio \& Pringle (1994) work.

\subsection{High State Spectra}

In spite of the lack of concurrent photometry we have concluded that ST LMi was likely accreting in the high state during our 2006-May 4-m spectra. ST LMi is often considered a "simple"

one-pole accretor, rather similar to VV Pup. There is general agreement in the literature regarding the geometry of the system: the orbital inclination is near $55^{\circ}$ and the main accreting pole has an inclination of $\sim 150^{\circ}$ with respect to the white dwarf spin axis (e.g. Schmidt, Stockman \& Grandi 1983; Cropper 1986; Peacock et al. 1992; Ferrario et al. 1993). This geometry leads to a self-eclipse of the main accretion region by the white dwarf, providing good leverage for extracting geometrical properties of this pole. The main accreting pole is the one nearest to the secondary star, having a magnetic field strength of $\sim 13 \mathrm{MG}$ ). The derived accretion footprint leads (in the direction of orbital motion) a line joining the stellar centers by $\sim 0.05-0.15$ phase units (Schmidt et al. 1983; Cropper 1988; Howell et al. 2000) being most directly viewed at our phase 0.8. The main accretion region has been shown to have a complex extended pattern on the white dwarf (e.g. Cropper \& Horne 1994; Stockman \& Schmidt 1996). The second pole has a strength of $\sim 30 \mathrm{MG}$ and has been 
reported to also accrete on occasion (Peacock et al. 1992).

Figure 10 presents a representative spectrum from the 2006-May-19 4-m run. $\mathrm{H} \alpha$ is strongly in emission with a complex and variable profile. Furthermore the HeI emission lines at $5876 \AA$ and $6678 \AA$ are quite strong, consistent with previous existing high state spectroscopy of the system, and inconsistent with the low state WIYN spectra in which the HeI emission lines are completely missing.

The phased high-state optical light curve of ST LMi (Stockman et al. 1983; Cropper 1986; Peacock et al. 1992; also see figure 4) has a bright phase lasting about 0.25 phase units centered near orbital phase 0.8 , in which the system becomes $\sim 1.2$ mag brighter in the V-band. The amplitude of this feature becomes larger as one moves from $\mathrm{B}$ to $\mathrm{V}$ to $\mathrm{R}$, consistent with our measurements. The bright phase corresponds to the time when the primary accreting pole is most directly viewed from the earth. The spectrum of ST LMi is significantly different between the bright orbital phases and the faint orbital phases of the high-state (see below). The high-state optical emission lines are quite complex and have varying interpretations involving infall velocities from an accretion funnel plus other components thought to be produced near the secondary star. (Schmidt, Stockman \& Grandi 1983; Bailey et al. 1985). This picture of the origin of the $\mathrm{H}$ and He emission lines during the high state bright phase is similar to that developed for other single pole accretors, such as VV Pup. In ST LMi, though, Schmidt et al. (1983) and Bailey et al. (1985) agree, as we discuss below, that some of the emission line components are from the secondary star and/or its vicinity.

Stockman et al. (1983) identified two major components in their ST LMi high-state optical emission lines ( $\mathrm{H} \delta, \mathrm{H} \gamma, \mathrm{HeI} 4471$, HeII 4686 and $\mathrm{H} \beta$ ): a broad emission component (BEC) which has a sinusoidal velocity curve with $\mathrm{K} \sim 500 \mathrm{~km} \mathrm{~s}^{-1}$ plus a non-sinusoidal narrow emission component (NEC) which is always redshifted with respect to the BEC. They conclude that the BEC emission arises near the primary star, in a cone-like structure having an infall velocity of $\sim 1000 \mathrm{~km} / \mathrm{sec}$. The high velocities imply that the emitting region should be close to the white dwarf, in a curved, organized path that leads to the observed line broadening. Using the same data, Schmidt et al. (1983) constructed a physical model for the accretion stream, and identified the BEC with a "constricted region" approximately mid-way between the two stellar components, where the accretion stream encounters strong magnetic pressure as well as becoming confined by the magnetic field of the white dwarf. The bright phase (0.5-0.95) NEC is attributed to a highly collimated infall of high-density gas, similar to the situation observed in EF Eri in its high state (Schneider \& Young 1980; Young et al. 1982). During the faint optical phase (0.0-0.5), the NEC (which dominates the peak of the emission) is thought to arise from the secondary star itself - possibly irradiation on the heated inner hemisphere. However, upon calculating the area that gives rise to this emission, these authors find that it is one order of magnitude less than the area of the irradiated inner hemisphere of the secondary star. This discrepancy is interpreted as being due to limb-darkening effects which were not taken into account in the calculation, and to a degree of self-eclipsing by the accretion funnel. 
The high state data of Bailey et al. (1985) found structure in the HeII $4686 \AA$ and $\mathrm{H} \beta$ emission lines similar to that reported in the Stockman et al. (1983) study. However, these authors argue that the profiles are made up of two sinusoids, one of which has variable width with phase. Component A appears narrow and reshifted for the bright part of the orbit, reaching velocities of $600 \mathrm{~km} / \mathrm{sec}$, whereas it is faint and broad at other phases. As in the work of Stockman et al. (1983) and Schmidt et al. (1983) this component is attributed to a highly curved inflow region onto the white dwarf. The second component (component B) has a sinusoidal velocity curve with $\mathrm{K} \sim 300 \mathrm{~km} \mathrm{~s}^{-1}$ and was attributed to the secondary star - either its irradiated inner hemisphere or material in its vicinity.

Our phase resolved profiles of the $\mathrm{H} \alpha$, HeI $5876 \AA$ and $\mathrm{HeI} 6678 \AA$ emission lines (figure 11) are complex, having many of the same features as shown in the earlier high-state studies of ST LMi. In particular, we see double-peaked emission lines between phases 0.6 and 0.9 , plus an extended blue wing for phases 0.1-0.4. The profiles are difficult to fit with multiple Gaussians because of their asymmetric nature plus the uncertainty regarding the number of components. For those reasons we chose to characterize the radial velocities using two simple methods: 1) the centroid of the full emission line, regardless of the structure, and 2) the velocities at the emission line peaks of one, or at most two, well-defined components. This is similar to the approach of Bailey et al. (1983), whereas Stockman et al. (1983) measured velocities of emission line components using bisectors and centroids. We find no significant differences in the shapes or velocities of the $\mathrm{He}$ and $\mathrm{H} \alpha$ emission lines.

In figure 12 (top) we show the measured radial velocities of the centroid of the full $\mathrm{H} \alpha$ emission line (open triangles) along with the velocities of the individual peaks. For comparison, we reproduce the velocity curve of the central $\mathrm{H} \alpha$ component from the low-state WIYN data (crosses). The other two panels include the measured RV of the centroid of the full HeI $5876 \AA^{\circ}$ (middle) and HeI $6678 \AA$ (bottom). In the top panel of fig. 12 we see that, for about half the orbit (phases 0.0-0.6), the centroid of the full $\mathrm{H} \alpha$ emission line (open triangles) follows the curve established by the 2006-Feb$21 / 22$ low state data (crosses), as does the radial velocity of the single peak of the line (solid points) over phases 0.0-0.6. However, over the phase interval 0.6-1.0 the centroid deviates sharply redward as do the two distinct emission line components (solid points). The redder component eventually deviates from the low-state curve by nearly $800 \mathrm{~km} \mathrm{~s}^{-1}$. The radial velocity of the peak of the blue component also deviates somewhat redward from the low-state curve. However, this is likely due to contribution of underlying emission from the redder component. We therefore will proceed under the assumption that the major change that occurs over phases 0.6-1.0 is the appearance of a new emission line component that is redshifted $700-800 \mathrm{~km} \mathrm{~s}^{-1}$ with respect to the persistant component.

Ferrario \& Wehrse (1999) have computed model line profiles for polars, finding a narrow, lowvelocity component from the magnetically heated coupling region, while a second component arises from inflow near the white dwarf. We think that the line component we observe over phases 0.6-1.0 is indeed due to inflow near the white dwarf, but identifying the "other" line component in our spectra as being from the coupling region does not seem to be possible. The magnetic field in ST 
LMi is similar to that in EF Eri, for which the distance of the coupling region above the white dwarf is estimated to be $5 \mathrm{R}_{W D}$ (Meggitt \& Wickramasinghe 1989). If the coupling region in ST $\mathrm{LMi}$ is also $\sim 5 \mathrm{R}_{W D}$, we find this emission to arise $\sim 0.13$ a above the white dwarf, while we find (using the system parameters from Stockman \& Schmnidt (1996) that the center of mass is $\sim 0.29$ a above the white dwarf. This is well on the white dwarf's side of the center of mass whereas the phasing of the "other" component in our spectra places the origin well on the secondary's side of the center of mass.

In figures 13 through 16 we present our high-state spectroscopy of ST LMi from 2006 May. The spectra are shown with assigned phases and each panel has the same identical flux scale. The spectra at phases $0.917,0.764$, and 0.865 correspond to the time of the orbital photometric bump, that is, the time when the accretion pole region is most nearly face-on to the observer (see figure 4 top, for comparison). These spectra have two features not seen at other orbital phases: 1) they have an absorption feature near $6275 \AA^{\circ}$ and 2) they have a broad continuum hump from $\sim 6400 \AA$ redward.

The broad absorption feature near $6275 \AA$ was also reported by Schmidt et al. (1983). Those authors considered several possibilities for the identification: $\lambda 6280 \mathrm{TiO}$ from the secondary star, the $\mathrm{H} \alpha \sigma^{-}$Zeeman absorption component from the white dwarf photosphere, and the telluric aband (due to $\mathrm{O}_{2}$ ). Their preferred identification was the $\mathrm{H} \alpha \sigma^{-}$line component from the white dwarf photosphere. Our improved spectral resolution allowed us to make a more careful comparison with the a-band. We used the very high resolution telluric spectrum above Kitt Peak from Hinkle, Wallace \& Livingston (2003), convolved with a Gaussian to match our spectral resolution. The match with the a-band is surprisingly good in wavelength, in approximate shape (degraded to the red in the average spectrum of phases $0.917,0.764$, and 0.865 ), and in approximate strength. Unlike water vapor however, oxygen is uniformly distributed in the earth's atmosphere and could not be modulated with phase to appear only over this small orbital phase range, even by coincidence. Like Schmidt et al. (1983), we too can rule out $\mathrm{TiO}$ for a similar reason: the $6275 \AA$ feature appears only during a small range in orbital phase. Both of these features occur only during the time when the observer has the most direct view of the white dwarf magnetic pole. Given the above, we also conclude that the $6275 \AA$ absorption feature is due to the $\mathrm{H} \alpha$ Zeeman $\sigma^{-}$line.

Examination of averaged WIYN low state spectra (figure 17) reveals the same Zeeman absorption component from $\mathrm{H} \alpha$ as we discussed above. The wavelength of the $\sigma^{-}$component can be used to calculate the surface magnetic field strength of the WD. Note, this field strength will be different from that estimated from the cyclotron humps as it comes from the surface while the cyclotron emission comes from somewhere in the column above the magnetic pole, probably, in fact a small range of locations. The separation of the Zeeman $\sigma^{-}$absorption component from the central $\pi$ component of $\mathrm{H} \alpha$ is $340 \AA$. Eq. 10 in Wickramasinghe \& Ferrario (2000) yields a B of 16.8 MG for this Zeeman separation in $\mathrm{H} \alpha$.

Looking at fig. 15 we see a redward hump in the continuum for those data obtained during the 
phase interval 0.7-0.9. The location of the hump corresponds to the $\mathrm{n}=14$ harmonic for a $\mathrm{B}=12 \mathrm{MG}$ field. This harmonic would be weak, if present at all. Cyclotron humps are far more prominent during times of very low mass accretion (e.g., Schmidt et al. 2005). We therefore argue that this redward rise in the continuum slope is not a cyclotron hump but the general cyclotron continuum (the Wien slope) observed during this time of high state mass accretion and seen during the interval of near face-on viewing of the accretion region.

The geometry of ST LMi's orbit as well as main accreting pole location allows a unique view of a magnetic CV. The accretion pole is not directly visible for long (or at all) and the binary inclination provides both a near-direct view as well as places the observer in a near perpendicular plane to a normal to the field lines at the pole. Thus, we can observe Zeeman absorption from the near pole white dwarf surface as well as the beamed cyclotron emission from the accretion column simultaneously. The fact that we do not see a direct pole or only for a small time period (phases 0.75-0.85), allows us to study the component stars during both high and low state with little contamination form the accretion flux.

\subsubsection{Hyperactivity on $C V$ secondary stars}

Magnetic activity on the mass-losing secondary star of CVs has been previously inferred from a variety of types of excess emission. Low state x-rays have been attributed to coronal emission from the secondary star in AM Her (de Martino et al. 1998), and ST LMi is one of a few systems for which chromospheric activity has been deduced from structures in the $\mathrm{H} \alpha$ emission line of lowstate optical spectra. There are currently only a handful of CVs whose low-state $\mathrm{H} \alpha$ profiles suggest chromospheric activity (hyperactivity). Apart from AM Her (Kafka et al. 2005b, 2006), VV Pup (Mason et al. 2007) and ST LMi, AR UMa (Schmidt et al. 1999) and TT Ari (Shafter et al. 1985) have been reported to show extra components in their low-state $\mathrm{H} \alpha$ emission line. Although these extra components in AR UMa and TT Ari have not been attributed to prominences on the secondary star, the emission line profiles and RV behavior are similar to the ones reported for AM Her and ST LMi. In all cases, the blue/red satellites are present for a portion of the orbit and, with the exception of ST LMi, the red satellite is present during the first part of the orbital cycle and the blue during the second part. In some instances both satellites are present at the same phases (triple-peaked emission lines), and in all cases there is a rapid switchover from red to blue satellite near spectroscopic conjunction. The persistence/duration of the satellites varies from system to system: in AM Her the satellites appear to have remained relatively unchanged for 3 years, the satellites in VV Pup appeared only during a second epoch of spectroscopic observations of the system and in ST LMi the visibility of the blue/red $\mathrm{H} \alpha$ satellites changes from cycle to cycle. Differences in the character and duration of activity is expected for different spectral types of secondaries and different rotation rates (i.e. system orbital periods).

It is also worth noting that the systems with reported hyperactivity are polars (diskless MCVs), with the exception of TT Ari. Although TT Ari is classified as a nova-like CV, Jameson et al. (1982) 
proposed that the system likely has a magnetic white dwarf therefore it belongs in the DQ Her category. An upper limit of $4 \mathrm{MG}$ (Shafter et al. 1985) for the magnetic field of the primary is still within the range of the magnetic field strength of the WDs in DQ Her (intermediate polar) systems. The suspected magnetic nature of TT Ari, along with the known magnetic nature of the other systems, suggests that a combination of fast rotation (all systems have orbital periods less than 4.0 hours) and magnetic field interaction between the two stellar components may be responsible for the presence of the satellites. Activity-induced line emission appears to be concentrated around the inner Lagrangian point, although the observed velocities suggest that the relevant structures are not confined to the orbital plane of the system. Again, an exception to this rule is presented by TT Ari, in which the $\mathrm{H} \alpha$ emission line appears to be stronger at inferior conjunction of the secondary star.

Triple-peaked H-alpha emission lines are not the only characteristic of stellar activity in CV secondaries: EF Eri, after spending seven years in a low state, and without changing its optical brightness, began showing weak and narrow Balmer lines and CaII H \& K emission, originating on or near the secondary star (Howell et al. 2006). Although there are indications that the EF Eri H $\alpha$ emission line shows additional components, the $\mathrm{S} / \mathrm{N}$ of the data was not adequate to reach secure conclusions about the structure of the emission line. On the other hand low states in disk systems are more rare than low states in MCVs. Chromospheric activity and activity cycles have been considered responsible for quasi-periodic modulations in the long-term optical light curves of dwarf nova in quiescence. Furthermore, variations (positive and negative) in the timings of eclipse minima in disk systems have been explained in terms of magnetic activity on the secondary (Baptista et al. 2003). Perhaps the most "direct" observation of stellar activity in a disk CV is provided by Steeghs et al. (1996) who described a peculiar stationary low velocity component in several emission lines of the quiescence spectra of the dwarf novae IP Peg and SS Cyg. The authors attributed this component to slingshot prominences (Collier Cameron \& Robinson 1989), co-rotating with the secondary star and kept in the vicinity of the L1 point by the binary potential. Taken at face value, this implies that the secondary stars of all CVs should be chromospherically active. Since the work of Parker (1961), it is known that a tachocline (convective zone - radiative core boundary) is necessary for the formation of a large scale, $\alpha \Omega$ (dipole) field, giving rise to all our familiar (from the sun) activity phenomena. Rotation can amplify the field giving rise to large-scale magnetic structures. Furthermore, magnetic field interactions may increase the lifetime of such structures. So far, the systems that show hyperactivity have secondary stars of spectral type M3 or later. Near that spectral type, main sequence stars become fully convective likely leading to a change in the nature of their magnetic field structure, because the formation of an $\alpha \Omega$-induced magnetic field is no longer possible. For fully convective stars, chromospheric emission may be generated by a small scale, local turbulent magnetic field (Durney et al. 1993). Although this might be an alternative to the $\alpha \Omega$ dynamo mechanism, it is not expected to produce large-scale features such as large starspots, or a dominant dipole field for magnetic braking. Other mechanisms might allow a fully convective star to produce a large scale field, such as an $\alpha^{2}$ dynamo (Chabrier and Kuker 2006). Fast rotation can amplify this field, which can easily reach the order of several kG, similar 
to the one that is observed in single active late $\mathrm{M}$ dwarfs, and similar to the one necessary for the observed activity on CV secondaries.

The He I emission line ratio is often used as an indicator of the physical conditions in the chromospheres of solar-type stars. It can be shown (Howell et al. 2006) that the ratio, $\mathrm{EW}[\mathrm{HeI}(5876 \AA)] / \mathrm{EW}[\mathrm{HeI}(6678 \AA)]$ can be as high as 45 in the quiet sun but is near 3 in active prominences. Using our 4-m high-state ST LMi spectra, we find that the ratio of $\operatorname{EW}[\operatorname{HeI}(5876 \AA)] / \operatorname{EW}[\operatorname{HeI}(6678 \AA)]$ emission lines is $\sim 2.5-3.0$, with the exception of phases $0.7-0.9$ when the accretion pole likely contributes to the line emission. This line ratio value is that expected for He I emission caused by collisional excitation in an active chromosphere hotter than $8000 \mathrm{~K}$.

\section{Final Remarks}

We have presented a spectroscopic and photometric study of ST LMi during its 2005-2006 high and low states. Our main results can be summarized as follows:

- In the low state, ST LMi remains at V 17.5 mag, with an occasional "hump" in the optical light curve, between phases 0.5 and 0.95 . This is the same phase interval during which the "bright" phase in the high state occurs due to the visibility of the accretion pole. Therefore episodic low-level accretion apparently takes place in ST LMi during the low state.

- The low-state spectra of the system are dominated by strong TiO bands from the secondary star. The only emission line is $\mathrm{H} \alpha$ and it appears to have structure similar that of AM Her (Kafka et al. 2005b, 2006) and VV Pup (Mason et al. 2007) in their low states. At times when accretion seems to have ceased completely (according to the optical light curves), the RVs of all components of $\mathrm{H} \alpha$ originate from the secondary's side of the center of mass. Concurrent photometry supports the lack of accretion and the presence of a large cool region (spot) near the L1 point (between phases 0.3-0.6).

- A photometric state $\sim 0.7$ mag fainter than the usual low state magnitude was observed. This is the second CV which displays such a state in its optical light curve. It is not clear whether this was a more complete cessation of mass transfer, or some other change in the system. It will be important to study such events if we to understand the properties of a CV in which accretion has truly stopped.

- In the high state, the $\mathrm{H} \alpha$ line presents a complicated multi-component profile. For orbital phases between 0.0 and 0.5 , the RV of the $\mathrm{H} \alpha$ emission line nearly coincides with the RVs of the low state $\mathrm{H} \alpha$ emission. This line component, which is phased with the motion of the secondary star, cannot be associated with accretion because it is present in both the high and low state. It is apparently not due to irradiation of the inner hemisphere of the secondary star because it is not brightest when the inner hemisphere most nearly faces the observer. It 
may be due to activity on the secondary star, although the geometry of the emission region is far from clear.

- In the high state a second emission line component of $\mathrm{H} \alpha$ appears near phase 0.8 which is highly red-shifted, likely due to infall onto the white dwarf. We confirm the results of Bailey et al. (1983) that a broad blue wing appears on the $\mathrm{H} \alpha$ profile at phases opposite the appearance of the highly red-shifted component.

Although CV secondaries are the fastest rotating lower main sequence stars known, the largescale activity signatures we have deduced are not expected for a fully convective star. It will be necessary to obtain suitable spectroscopy of a non-magnetic (disk) CV in the low state to test if the presence of $\mathrm{H} \alpha$ satellites are tied to magnetic field interactions of the two stars, or if ultra-fast rotation along suffice for their presence.

We would like to thank our anonymous referee for the careful review of the manuscript.

\section{REFERENCES}

Bailey, J., et al. 1985, MNRAS, 215, 179

Baptista, R., Borges, B. W., Bond, H. E., Jablonski, F., Steiner, J. E., \& Grauer, A. D. 2003, MNRAS, 345, 889

Bianchini, A. 1988, Informational Bulletin on Variable Stars, 3136, 1

Chabrier, G., Kumlker, M. 2006, A\&A, 446, 1027

Collier Cameron, A., \& Robinson, R. D. 1989, MNRAS, 238, 657

Cropper, M. 1986, MNRAS, 222, 853

Cropper, M., \& Horne, K. 1994, MNRAS, 267, 481

de Martino, D., et al. 1998, A\&A, 333, L31

Durney, B. R., De Young, D. S., \& Roxburgh, I. W. 1993, Sol. Phys., 145, 207

Ferrario, L., Bailey, J., \& Wickramasinghe, D. T. 1993, MNRAS, 262, 285

Ferrario, L., \& Wehrse, R. 1999, MNRAS, 310, 189

Hinkle, K. H., Wallace, L., \& Livingston, W. 2003, Bulletin of the American Astronomical Society, 35,1260

Henden, A. A., \& Honeycutt, R. K. 1995, PASP, 107, 324

Howell, et al., 2006, ApJ, 652, 709.

Howell, S. B., Ciardi, D. R., Dhillon, V. S., \& Skidmore, W. 2000, ApJ, 530, 904 
Jameson, R. F., Sherrington, M. R., King, A. R., \& Frank, J. 1982, Nature, 300, 152

Kafka, S., Robertson, J., Honeycutt, R. K., \& Howell, S. B. 2005, AJ, 129, 2411 (2005a)

Kafka, S., Honeycutt, R. K., Howell, S. B., \& Harrison, T. E. 2005, AJ, 130, 2852 (2005b)

Kafka, S., Honeycutt, R. K., \& Howell, S. B. 2006, AJ, 131, 2673

Kafka, S., \& Honeycutt, R. K. 2005, AJ, 130, 742 [KH05]

King, A. R., \& Cannizzo, J. K. 1998, ApJ, 499, 348

Livio, M., \& Pringle, J. E. 1994, ApJ, 427, 956

Linnell, A.P., Szkody, P., Gansicke, B., Long, K. S., Sion, E. M., Hoard, D. W., \& Hubeny,I. 2005, ApJ, 624, 923

Mason et al. 2007, A\&A in press

Meggitt, S. M. A., \& Wickramasinghe, D. T. 1989, MNRAS, 236, 31

Peacock, T., Cropper, M., Bailey, J., Hough, J. H., \& Wickramasinghe, D. T. 1992, MNRAS, 259, 583

Shafter, A. W., Szkody, P., Liebert, J., Penning, W. R., Bond, H. E., \& Grauer, A. D. 1985, ApJ, 290,707

Schmidt, G. D., Stockman, H. S., \& Grandi, S. A. 1983, ApJ, 271, 735

Schmidt, G. D., Hoard, D. W., Szkody, P., Melia, F., Honeycutt, R. K., \& Wagner, R. M. 1999, ApJ, 525, 407

Schmidt, G. D., et al. 2005, ApJ, 630, 1037

Schneider, D. P., \& Young, P. 1980, ApJ, 238, 946

Steeghs, D., Horne, K., Marsh, T. R., \& Donati, J. F. 1996, MNRAS, 281, 626

Stockman, H. S., Foltz, C. B., Schmidt, G. D., \& Tapia, S. 1983, ApJ, 271, 725

Stockman, H. S., \& Schmidt, G. D. 1996, ApJ, 468, 883

Warner, B. 1995, Cambridge Astrophysics Series, Cambridge, New York: Cambridge University Press, -c1995,

Wickramasinghe, D. T., \& Ferrario, L. 2000, PASP, 112, 873

Young, P., Schneider, D. P., Sargent, W. L. W., \& Boksenberg, A. 1982, ApJ, 252, 269 
Table 1. Log of Observations of ST LMi

\begin{tabular}{ccccc}
\hline \hline UT Date & Seq. No. & Telescope/Instrument & No. of Exposures/Filter & Exp time(s) \\
\hline PHOTOMETRY: & & & \\
1991-01 to 2005-03 & & RoboScope 0.4-m & $551 / \mathrm{V}$ & 240 \\
$2005-02-28$ & A1 & WIYN 0.9-m/S2KB & $44 / \mathrm{V}$ & 120 \\
$2005-03-01$ & A2 & WIYN 0.9-m/S2KB & $35 / \mathrm{R}$ & 90 \\
$2005-03-02$ & A3 & WIYN 0.9-m/S2KB & $42 / \mathrm{V}$ & 90 \\
$2005-03-03$ & A4 & WIYN 0.9-m/S2KB & $50 / \mathrm{V}$ & 90 \\
$2005-03-04$ & A5 & WIYN 0.9-m/S2KB & $33 / \mathrm{V}$ & 90 \\
$2005-06-13$ & B1 & WIYN 0.9-m/S2KB & $11 / \mathrm{V}$ & 180 \\
$2005-06-17$ & B2 & WIYN 0.9-m/S2KB & $12 / \mathrm{V}$ & 120 \\
$2005-06-20$ & B3 & WIYN 0.9-m/S2KB & $13 / \mathrm{V}$ & 120 \\
$2005-12-23$ & & Tenagra 0.8-m & $1 / \mathrm{V}$ & 200 \\
$2005-12-24$ & & Tenagra 0.8-m & $23 / \mathrm{V}$ & 200 \\
$2006-02-12$ & C1 & WIYN 0.9-m/S2KB & $6 / \mathrm{V}$ & 120 \\
$2006-02-21$ & & WIYN 0.9-m/S2KB & $68 / \mathrm{V}$ & 180 \\
$2006-02-22$ & D1 & WIYN 0.9-m/S2KB & & 180 \\
SPECTROSCOPY: & & WIYN 3.5-m/Hydra & 6 & $600-1800$ \\
$2005-06-17$ & & KPNO 4-m/RC Spec & 2 & 1200 \\
$2005-12-31$ & & WIYN 3.5-m/Hydra & 12 & 600 \\
$2006-02-21$ & & WIYN 3.5-m/Hydra & 31 & 600 \\
$2006-02-22$ & & KPNO 4-m/RC Spec & 16 & $300-600$ \\
$2006-05-19$ & & &
\end{tabular}



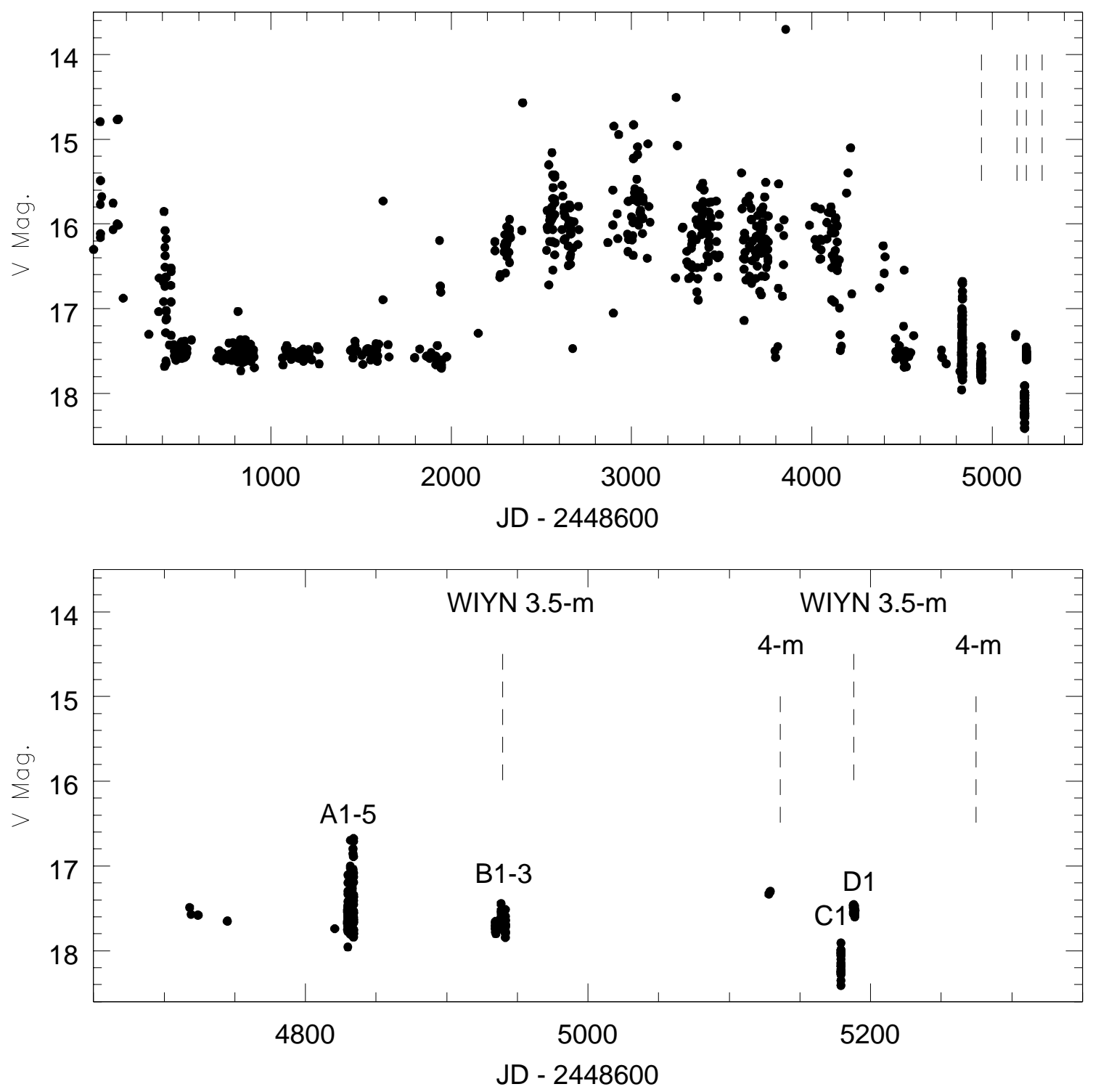

Fig. 1. - Top: V-band light curve of ST LMi 1990-2005 from various sources, mostly RoboScope. The dates of our spectroscopy are noted as vertical dashed lines. Bottom: Expanded view of the latter portion of the top panel, with the spectroscopic observing runs noted. The labeled photometric sequences correspond to the designations in figure 2 and Table 1. 

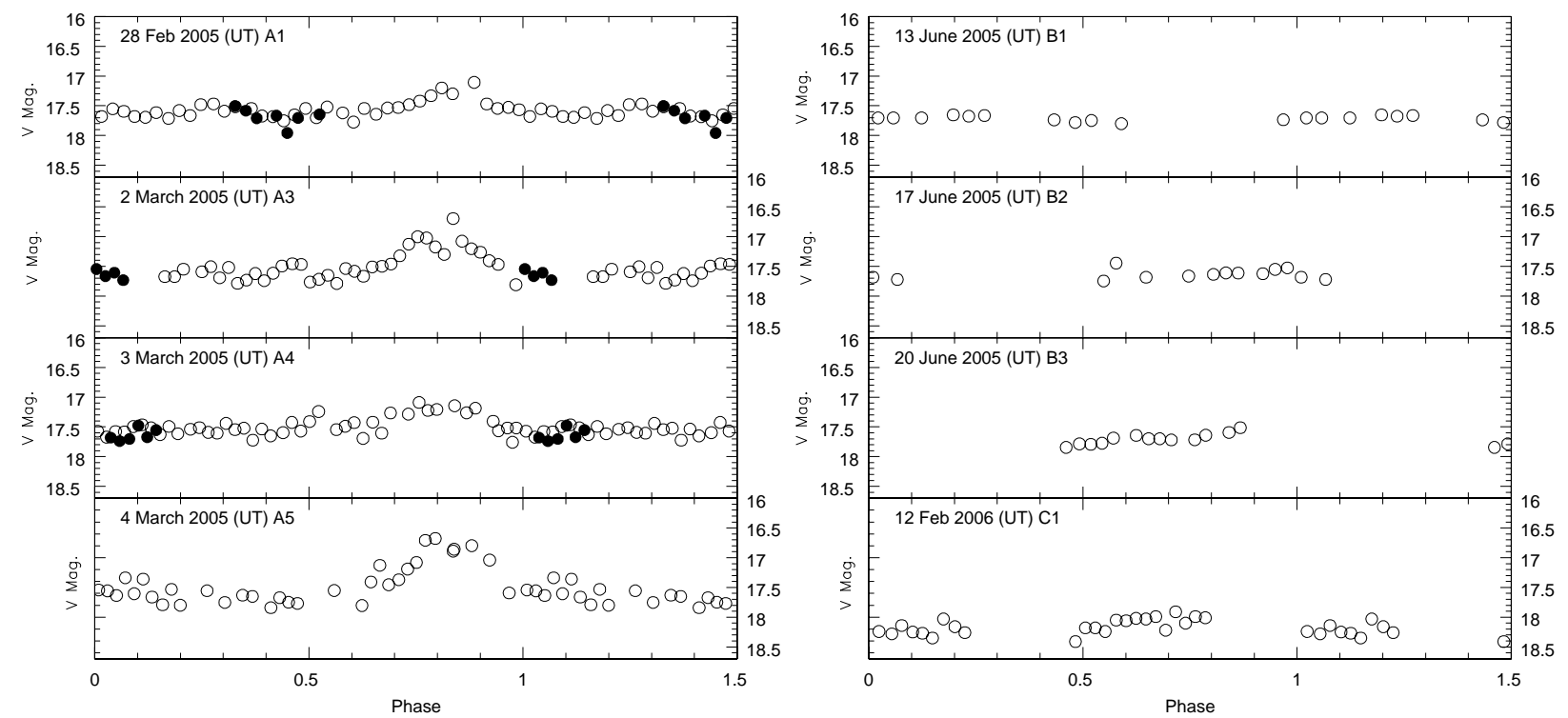

Fig. 2.- Left: Phased V-band light curves of ST LMi from 4 nights of observing run A, as marked in figure 1 (bottom). Note the maxima near phase 0.8. Filled symbols are data from the second of two adjacent orbital cycles. Right: Phased V-band light curves of ST LMi from 3 nights of observing run $\mathrm{B}$ and 1 night of observing run $\mathrm{C}$, as marked in figure 1 (bottom). Note the absence of a maxima near phase 0.8 

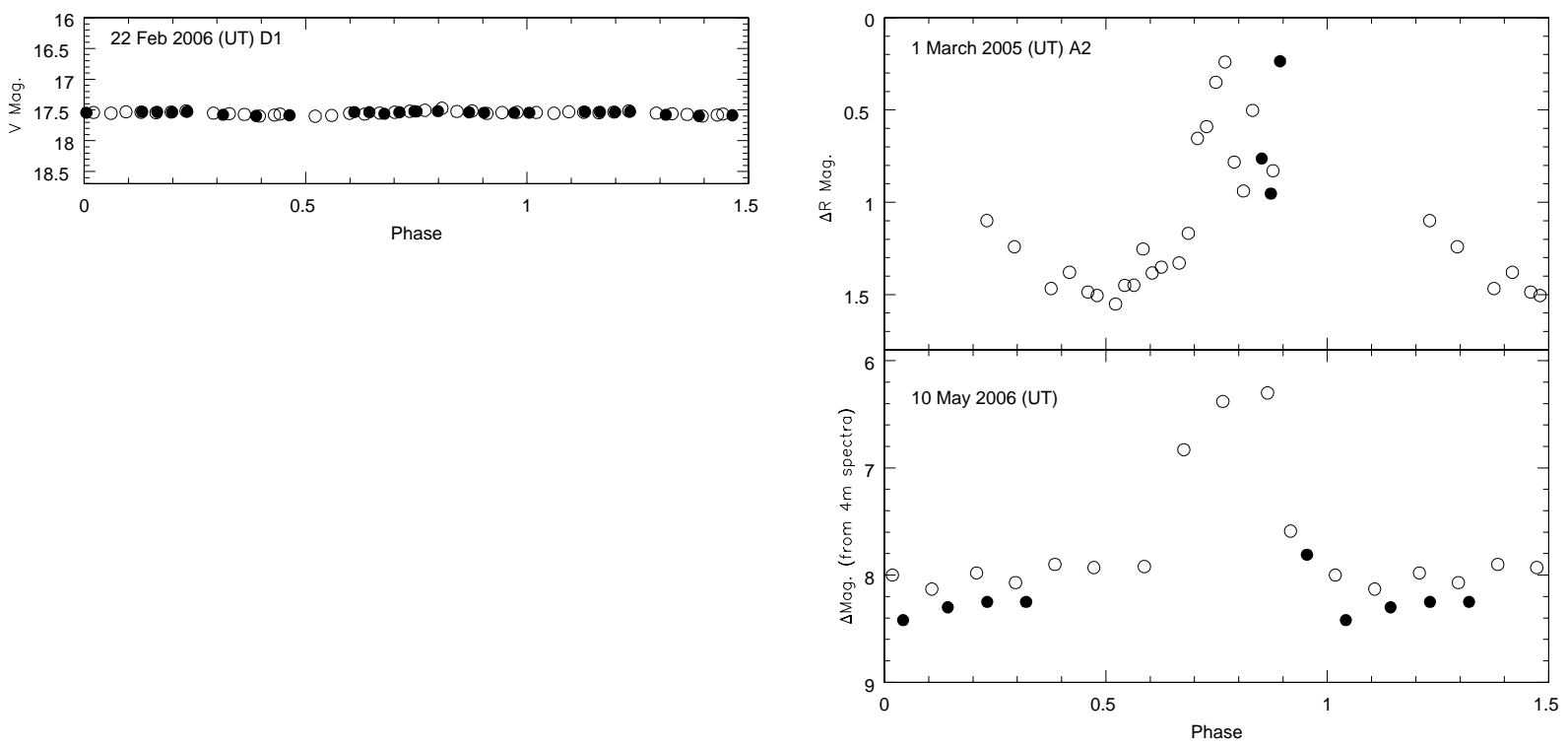

Fig. 3. - Left: Phased light curve of ST LMi from observing run D, as marked in figure 1 (bottom). Note the weakness of the maximum near phase 0.8 compared to figure 2 (left), and the near absence of scatter in the light curve compared to figure 2 and figure 4 (bottom). Filled symbols are data from the second of two adjacent orbital cycles. Right: Phased R-band light curves of ST LMi. Top is a differential R-band light curve from run A2, as marked in figure 1 (bottom). Bottom is a synthetic light curve extracted from the 4-m spectra of 2006-May-19, with an effective wavelength approximately midway between $\mathrm{V}$ and $\mathrm{R}$. Note the large amplitude of the maximum near phase 0.8 in both plots. 


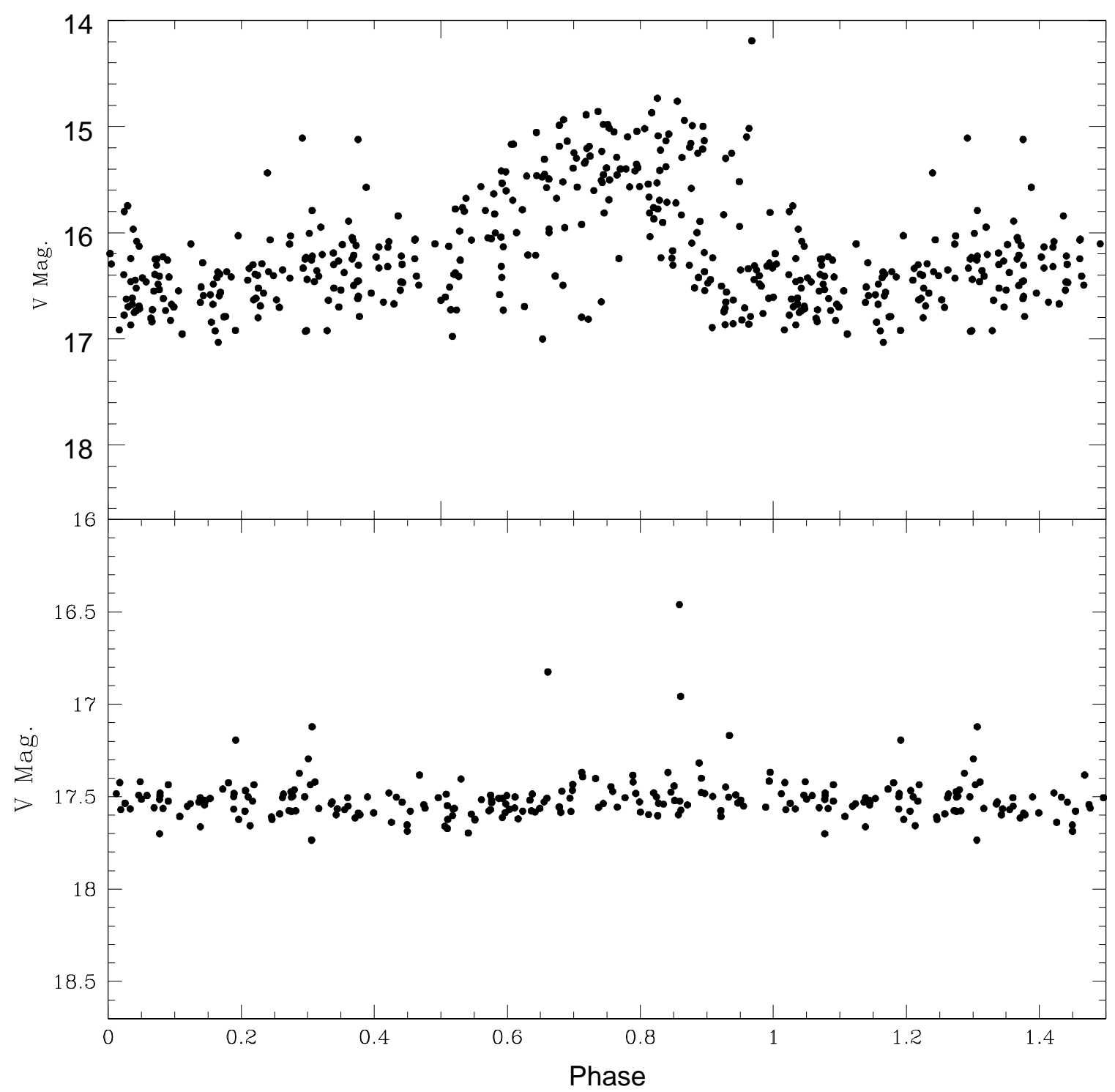

Fig. 4. - Phased V-band light curves of ST LMi from RoboScope data 1990-2005. The data consist of single point each clear night, at a random orbital phase. Top is the high state light curve showing a maximum near phase 0.8. Bottom is the low-state light curve, perhaps showing a weak maximum near phase 0.8 . The occasional brightward excursions in the bottom panel are likely artifacts due to cosmic rays. 


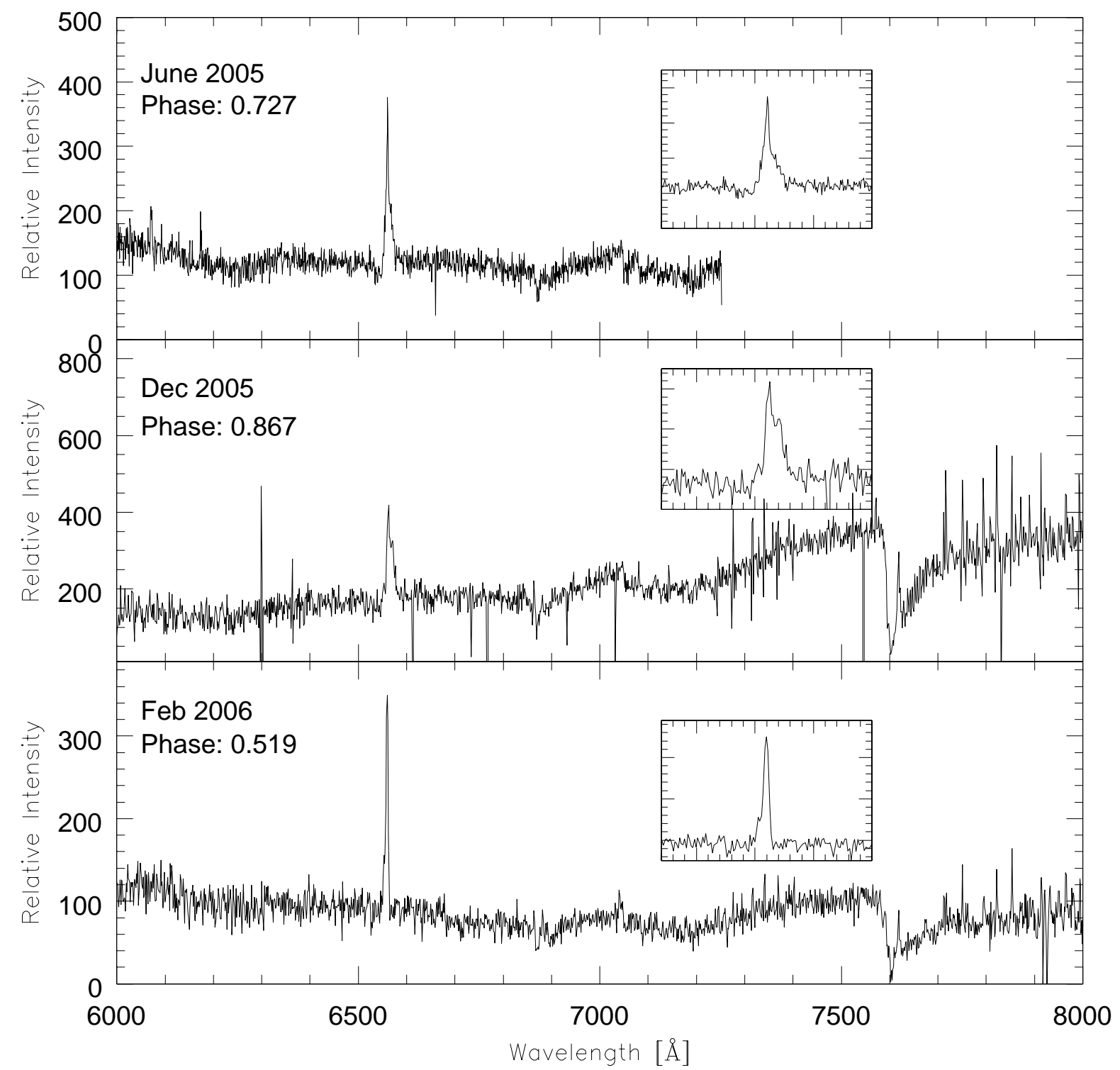

Fig. 5.- Representative low-state spectra of ST LMi from 2005-Jun (top), 2005-Dec (middle) and 2006-Feb (bottom). No corrections for atmospheric absorption have been made. The middle panel shows sharp features due to incomplete cancellation of night sky emission lines. The inset plots show the $\mathrm{H} \alpha$ emission line on an expanded scale. 

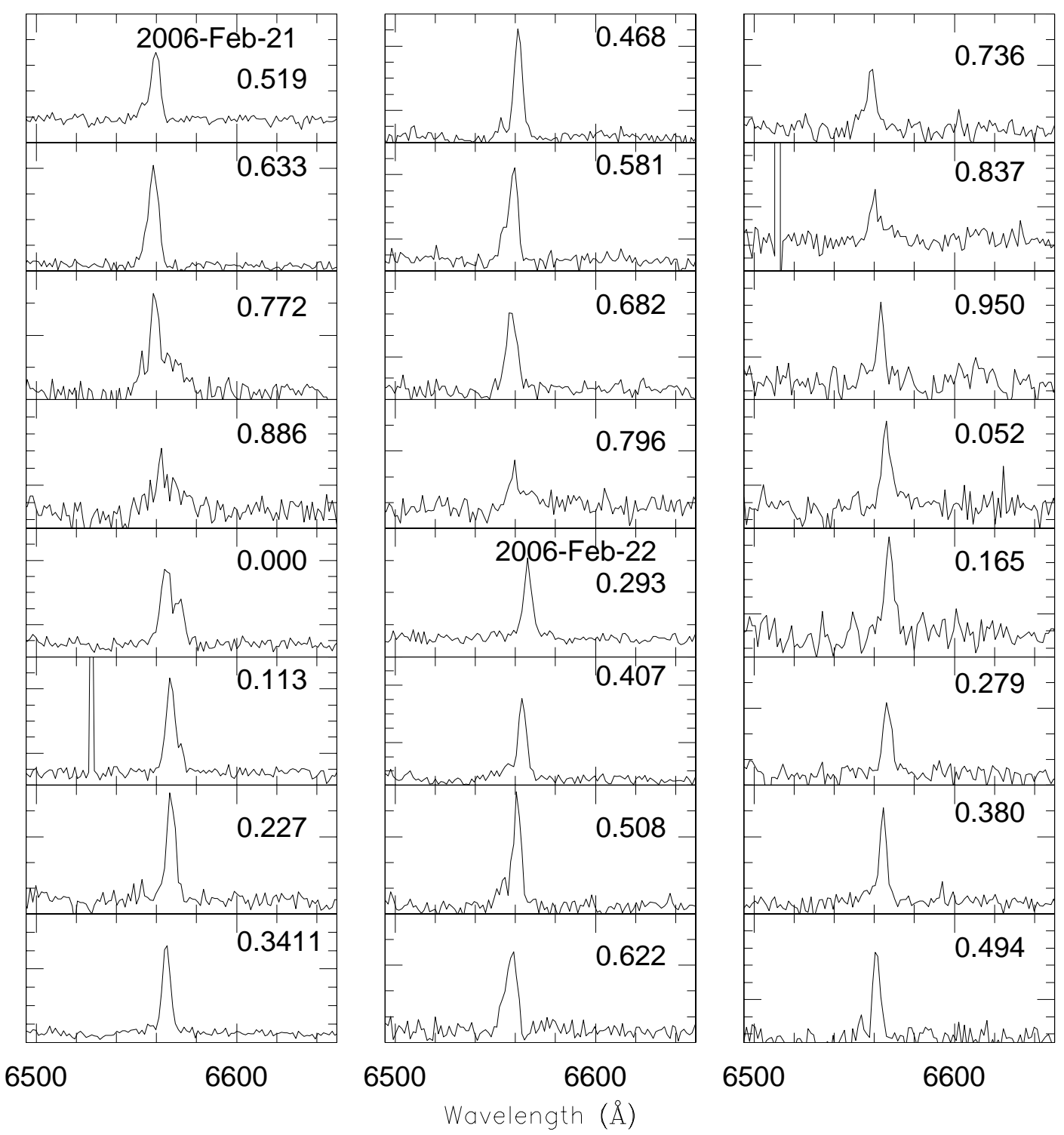

Fig. 6. - Low-state $\mathrm{H} \alpha$ profiles of ST LMi for orbital phases 0.5192 to 0.7963 on 2006-Feb-21 UT (first night) and for phases 0.2930 to 0.4942 on 2006-Feb-22 UT (second night)). Successive phases run down the page, starting in the upper left corner. 

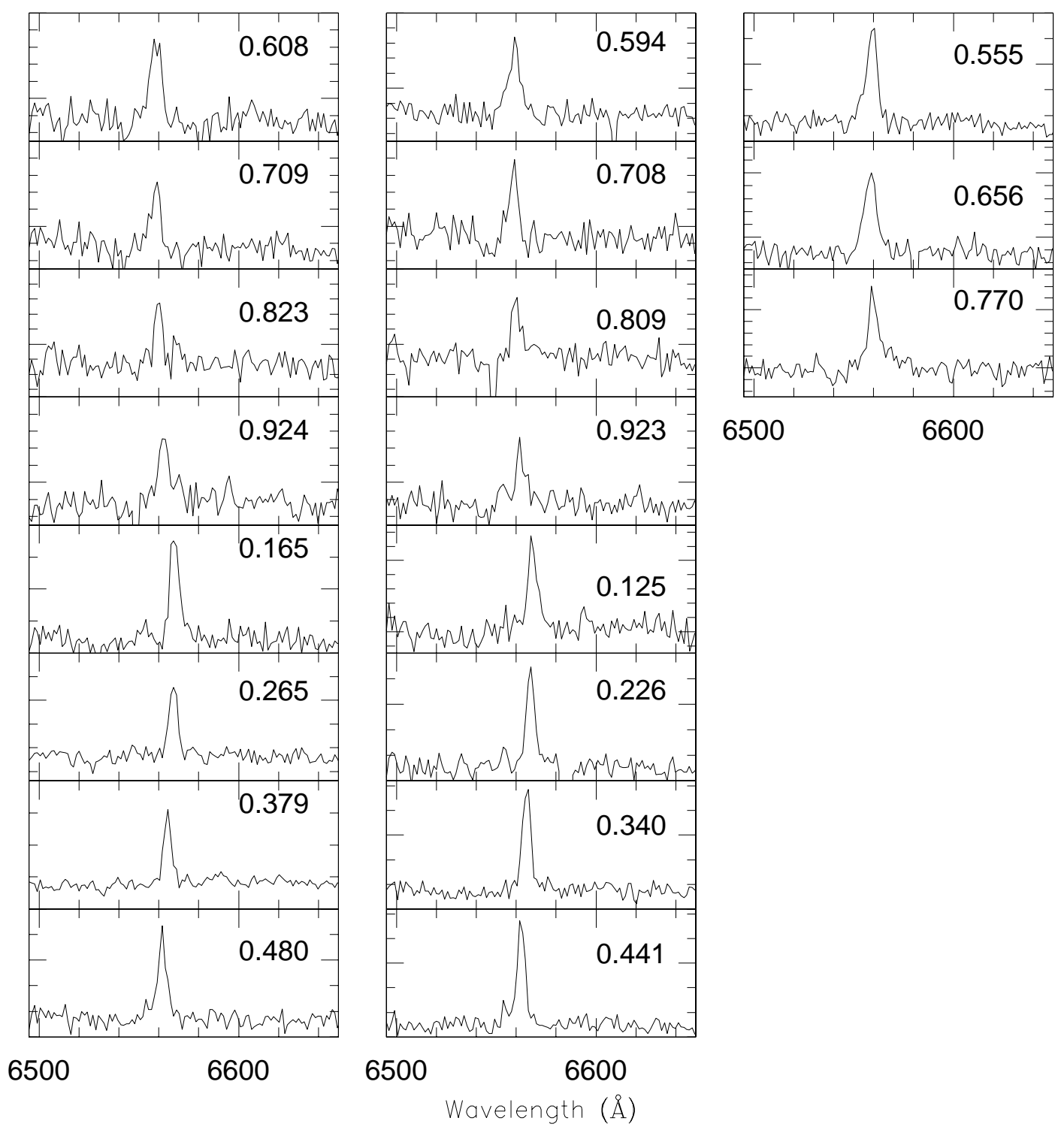

Fig. 7.- Continuation of previous figure (for night 2). 


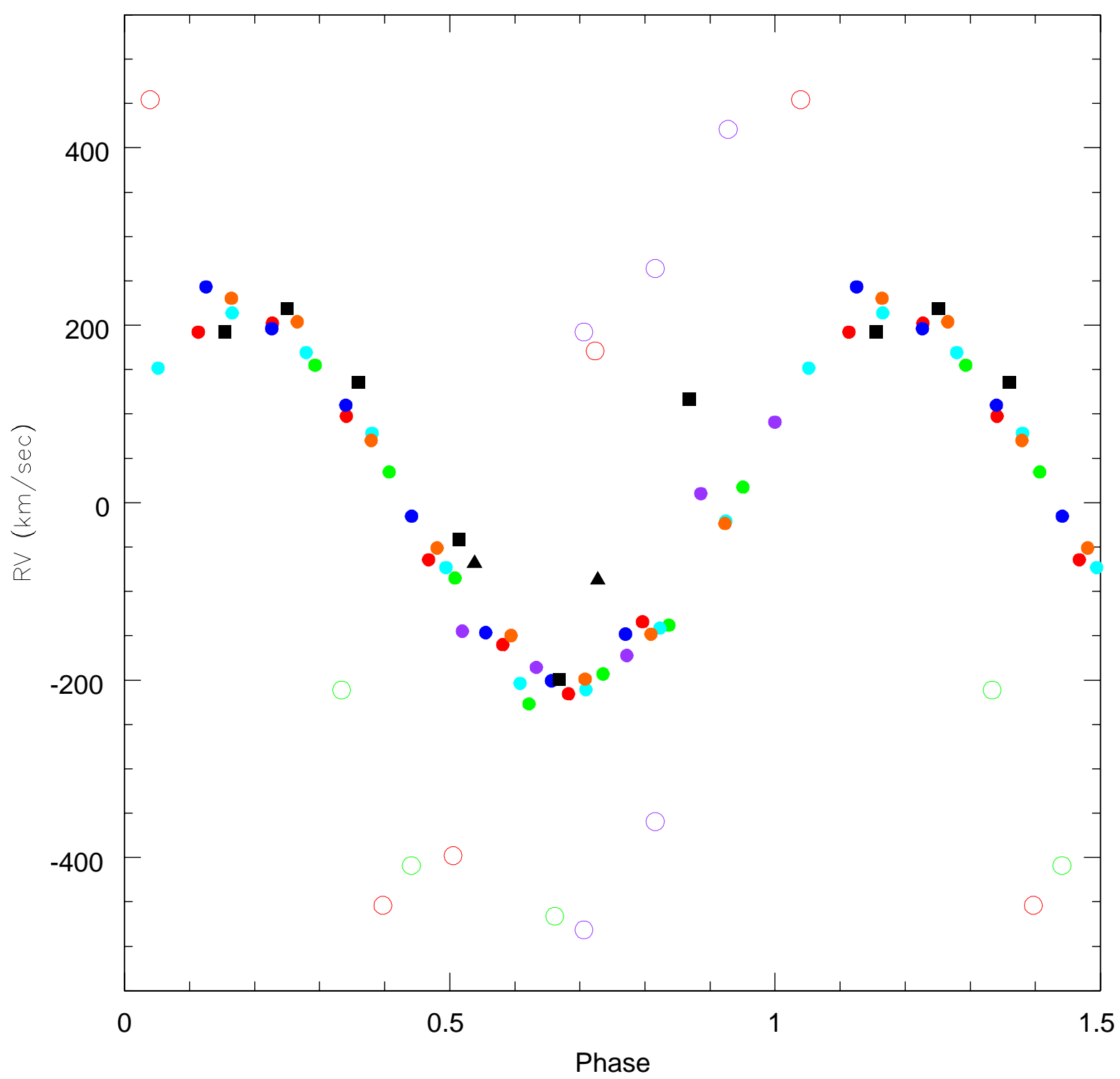

Fig. 8. - The RVs of the central $\mathrm{H} \alpha$ emission line component in ST LMi for the low-state WIYN spectra of 2005-June-17 (squares), the 4-m spectra of 2005-Dec-31 (triangles), and the low-state WIYN spectra of 2006-Feb-21/22 (solid points). Open circles represent the RVs of the occasional satellite components in the WIYN 2006-Feb spectra. Colors represent points of the same orbit. 


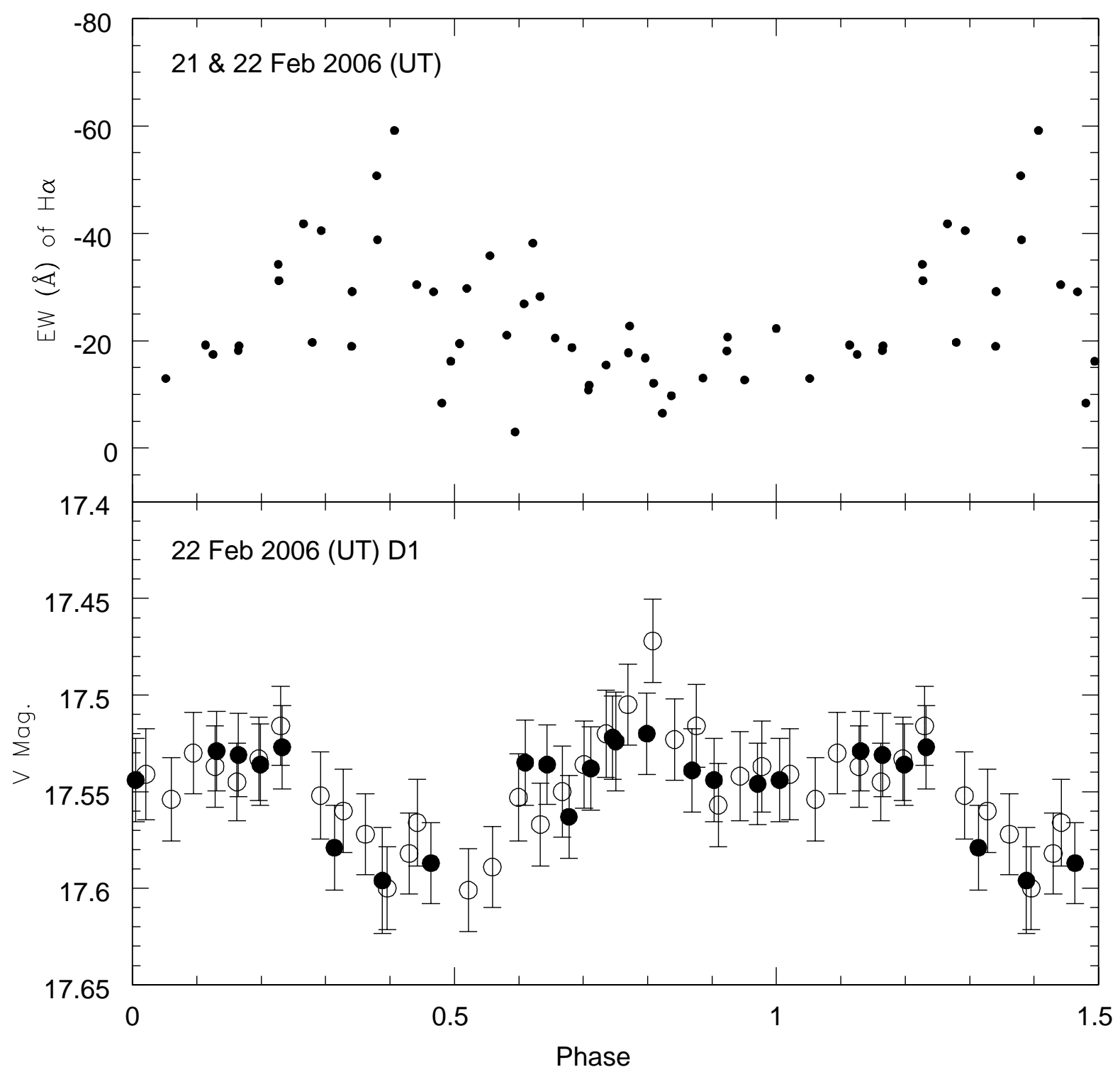

Fig. 9.- Top: Total EW of the low-state WIYN H $\alpha$ emission line vs orbital phase; Bottom: The concurrent phase light curve. 


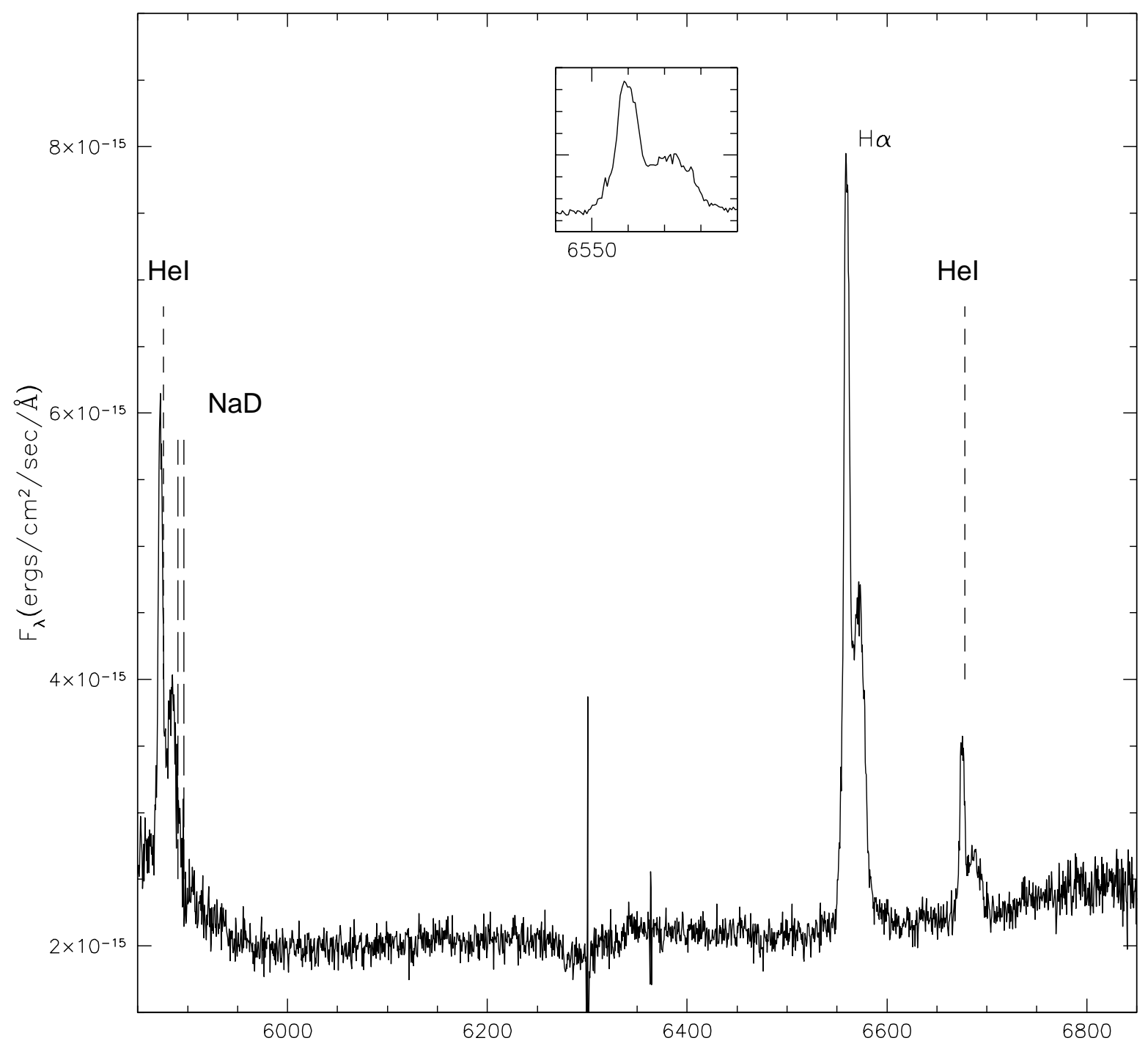

Fig. 10. - A representative high-state spectrum of ST LMi from the 2006-May-19 (UT) 4-m run. The inset plot is an expanded view of the $\mathrm{H} \alpha$ profile. 

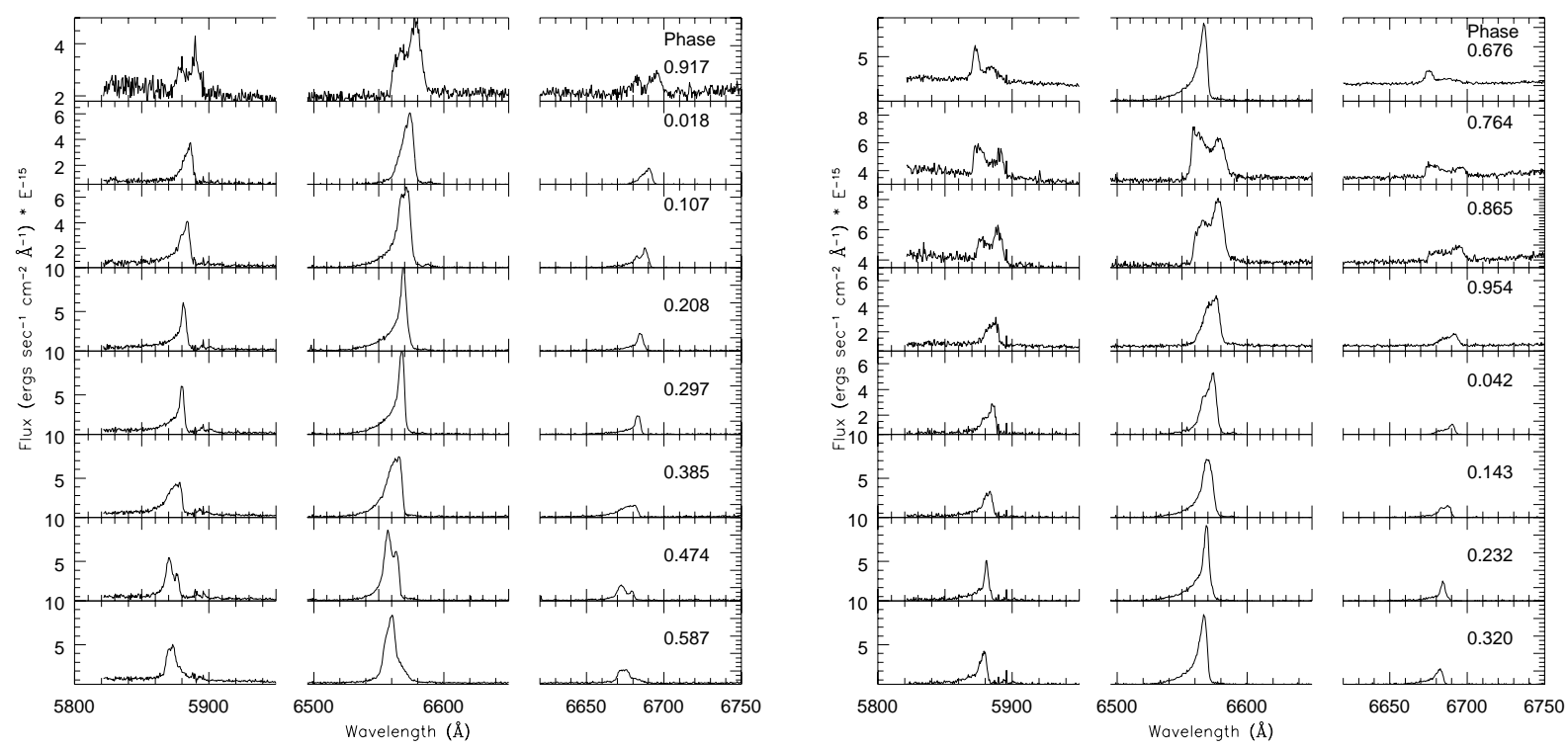

Fig. 11. - Line profiles of HeI 5876 $\AA$ (left column), H $\alpha$ (center column) and HeI $6678 \AA$ (right column) as a function of orbital phase, for the high-state spectra of 2006-May-19 2006 UT. The $\mathrm{NaD}$ emission lines (from Tucson airglow) are often visible near and sometimes within the red wing of HeI $5876 \AA$. 


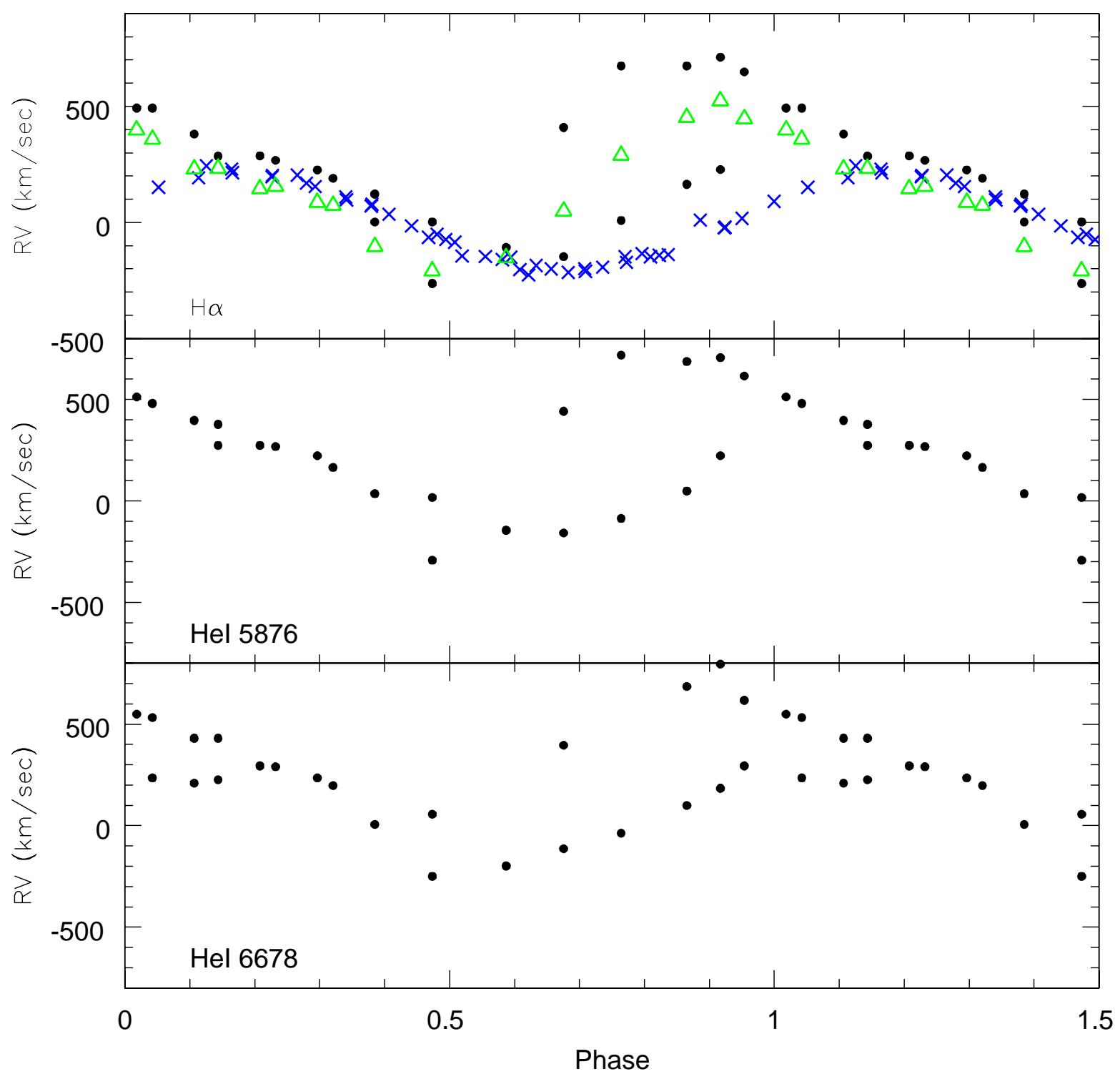

Fig. 12. - High-state (2006-May-19 UT) radial velocity curves of ST LMi for H $\alpha$ (top), and two HeI emission lines (middle and bottom). The open triangles are velocities of the centroid of $\mathrm{H} \alpha$. The filled circles are velocities at the peaks of the most distinct one or two emission line components, as seen in the line profiles in figure 10. For comparison, the crosses in the top panel are the ST LMi $\mathrm{H} \alpha$ velocities for the low-state WIYN data of 2006-Feb-21/22 UT. 


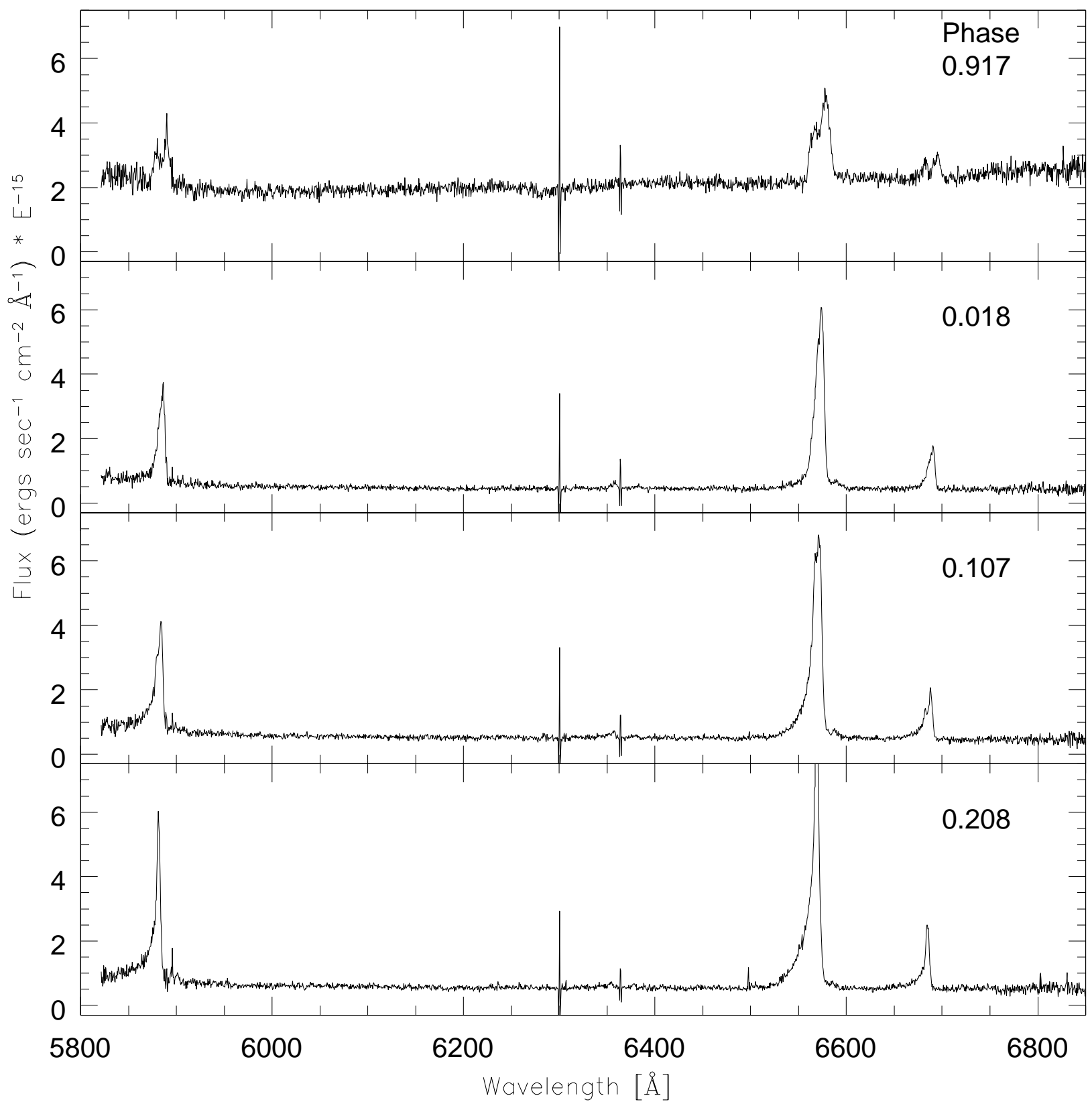

Fig. 13. - The complete spectral region of ST LMi as a function of orbital phase for the high-state 2006-May-19 (UT) data. We are using the same scale for all spectra, to emphasize the continuum and the weaker spectral features. (See text for discussion.) 


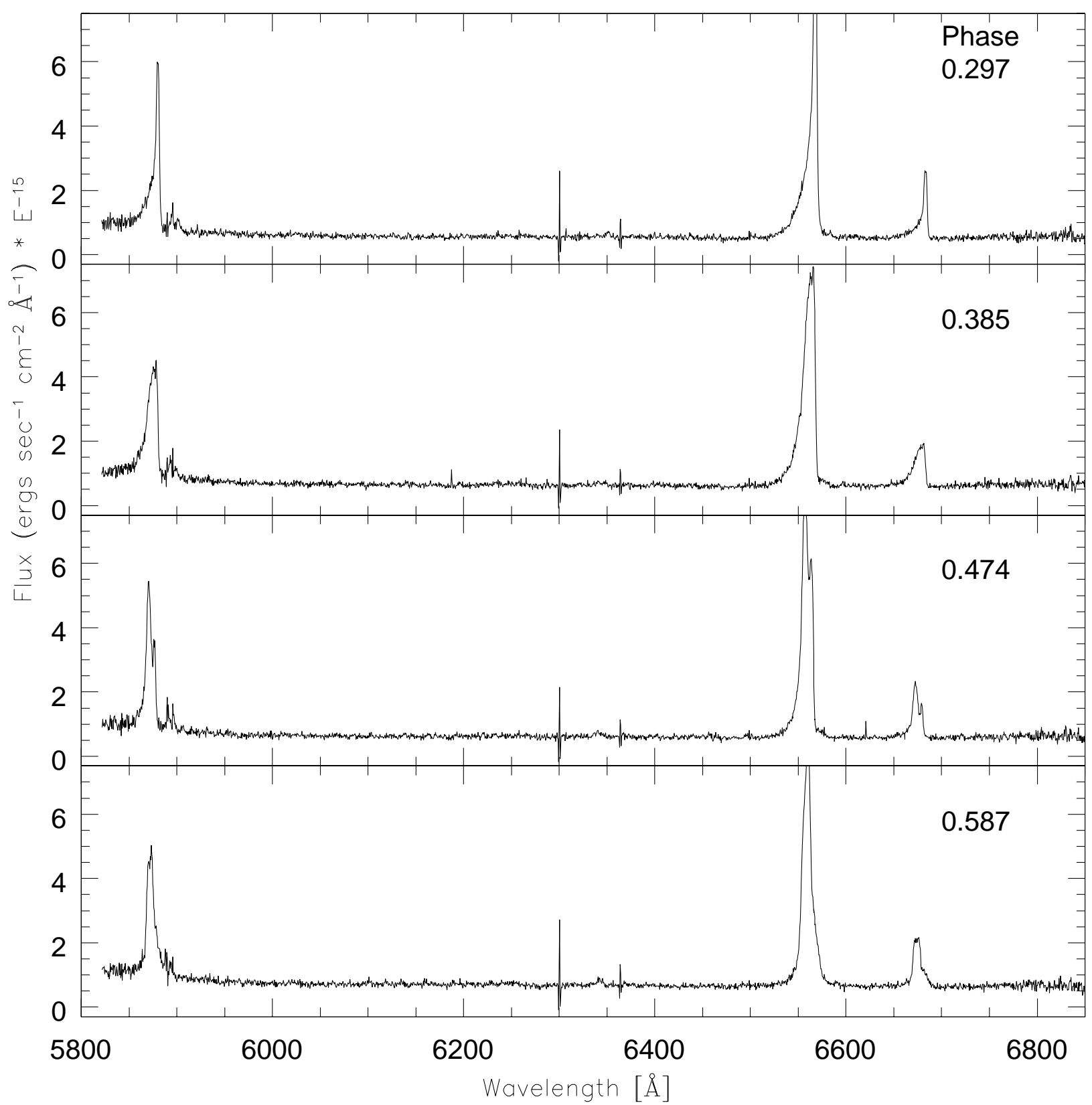

Fig. 14. - The complete spectral region of ST LMi as a function of orbital phase for the high-state 2006-May-19 (UT) data (cont). 


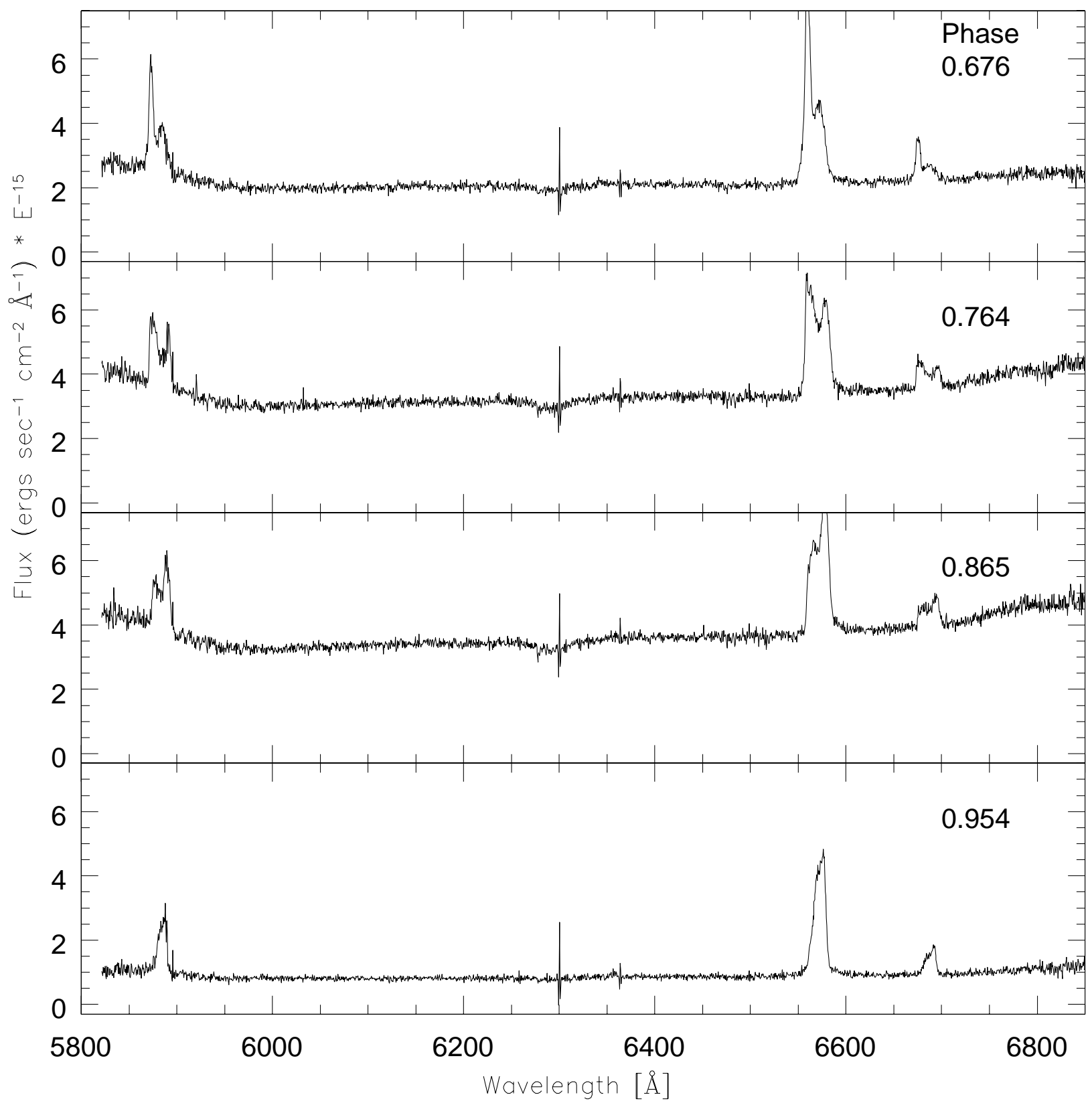

Fig. 15. - The complete spectral region of ST LMi as a function of orbital phase for the high-state 2006-May-19 (UT) data (cont) 


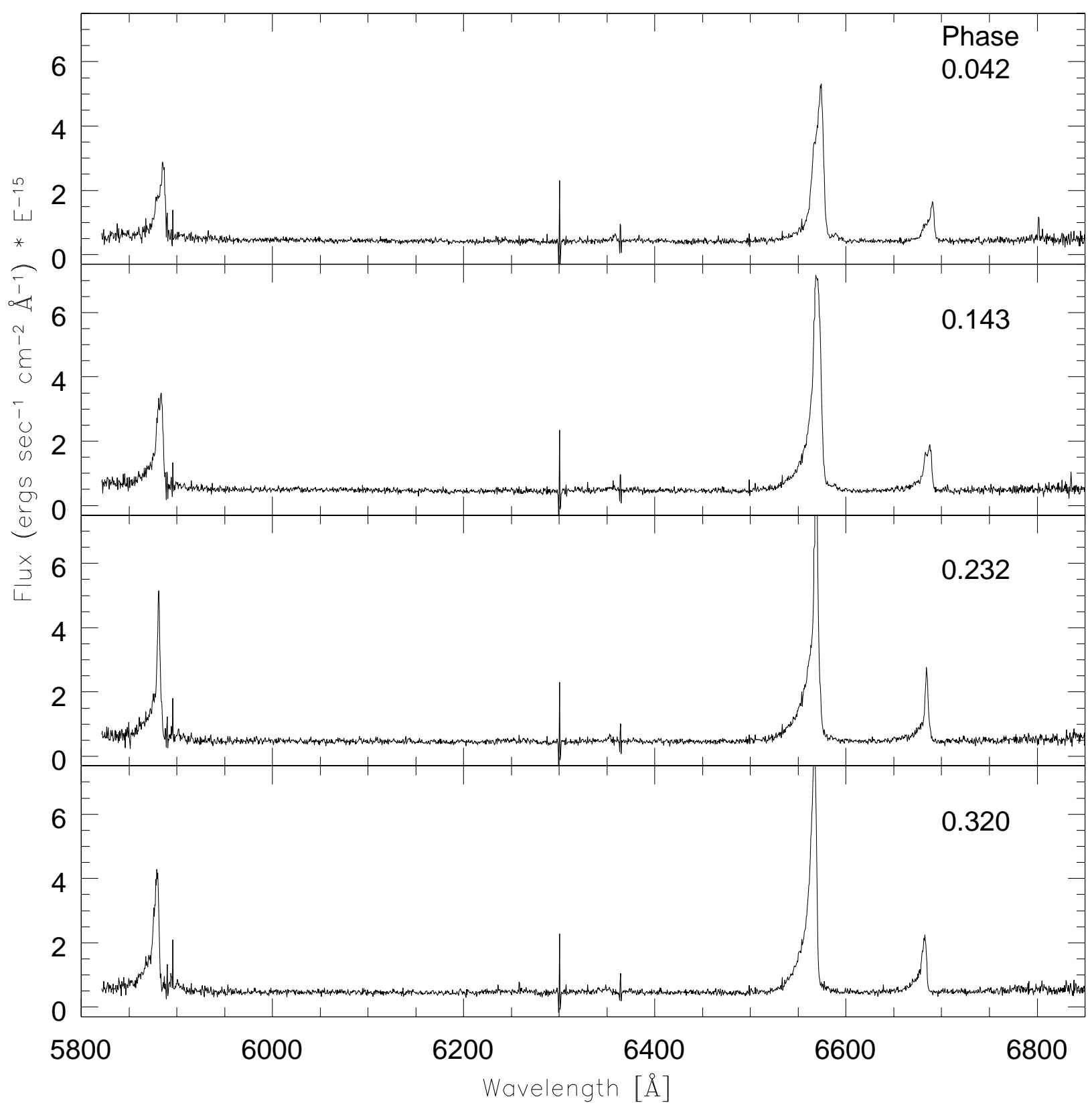

Fig. 16. - The complete spectral region of ST LMi as a function of orbital phase for the high-state 2006-May-19 (UT) data (cont) 

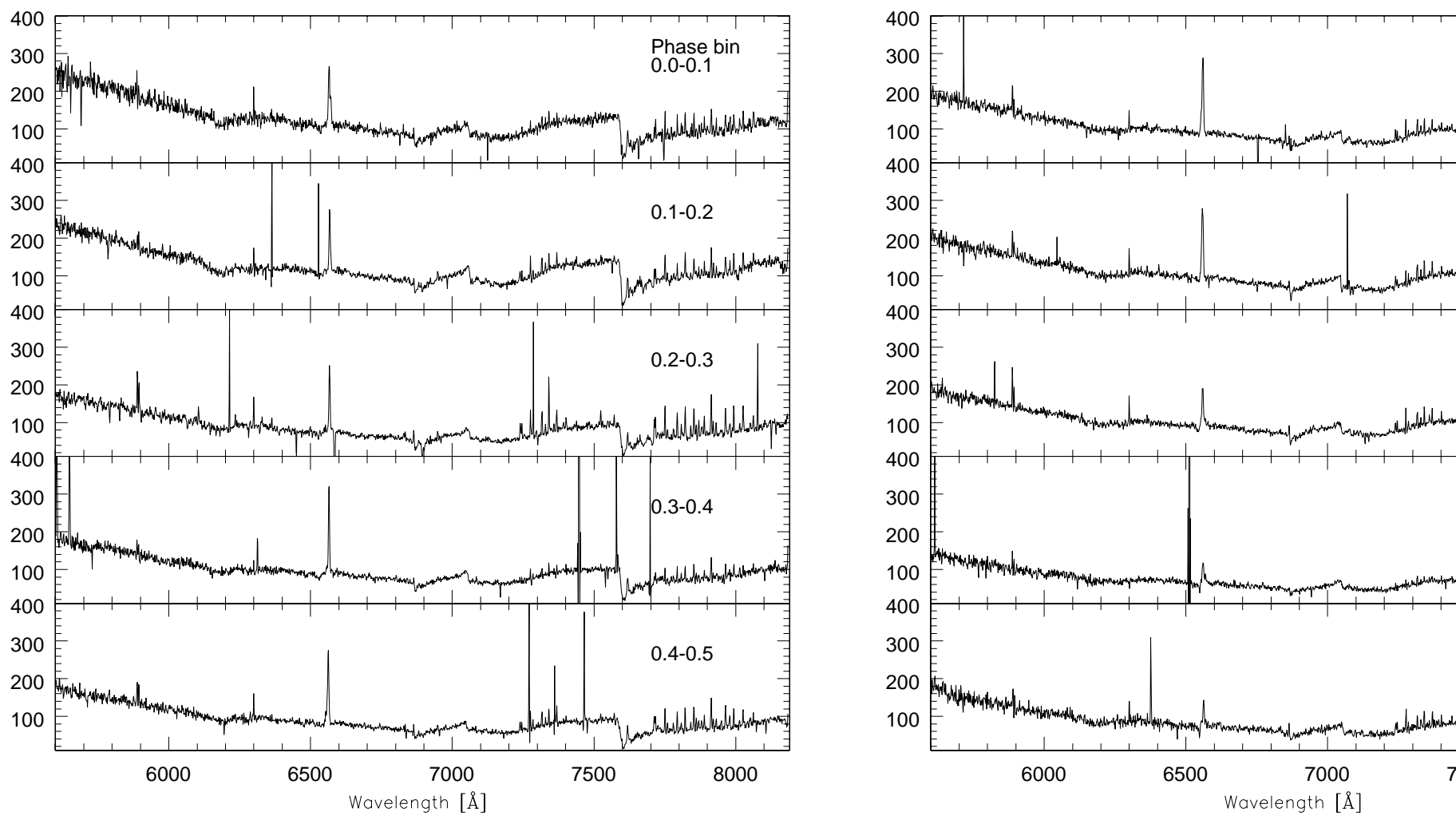

Fig. 17.- WIYN low-state spectra from 2006-Feb-21/22 UT, averaged in phase bins of 0.1. The narrow weak emission lines, mostly redward of $7200 \AA$, are artifacts due to incomplete cancellation of $\mathrm{OH}$ features in the night sky from the fiber spectra. (Also see text for comments.) 